\title{
History of Magnetism and Basic Concepts
}

\author{
J. M. D. Coey $\mathbb{1 0}$
}

\section{Contents}

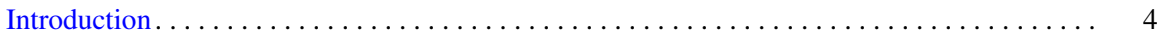

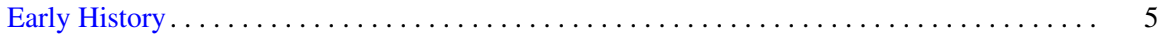

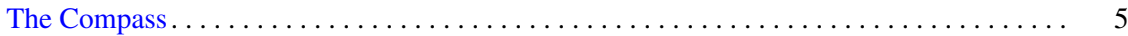

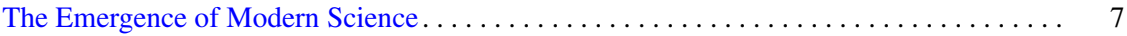

The Electromagnetic Revolution $[9] \ldots \ldots \ldots \ldots \ldots \ldots \ldots \ldots \ldots \ldots \ldots \ldots \ldots \ldots \ldots \ldots . \ldots \ldots$

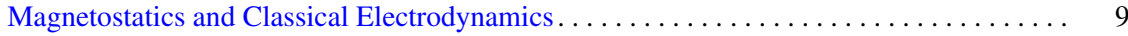

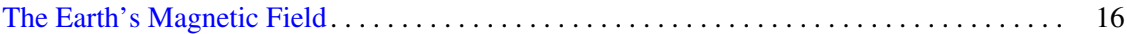

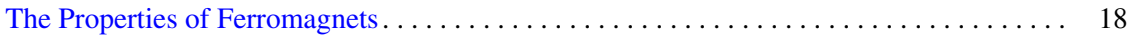

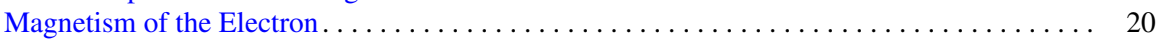

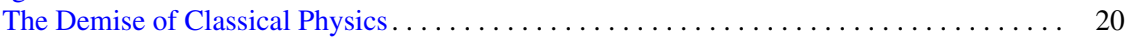

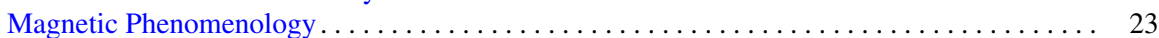

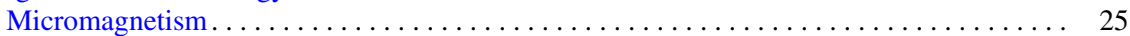

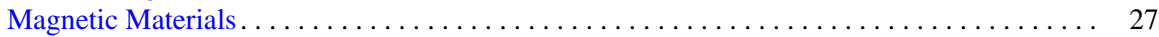

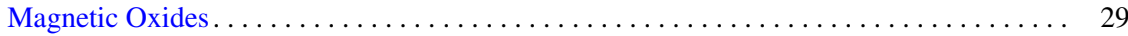

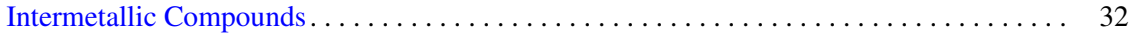

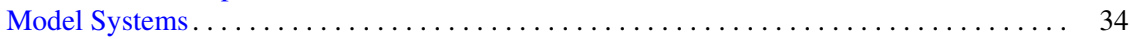

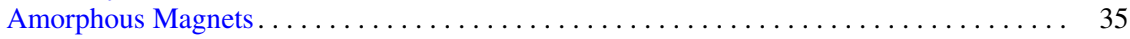

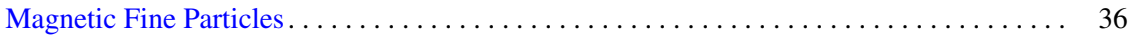

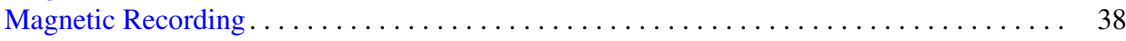

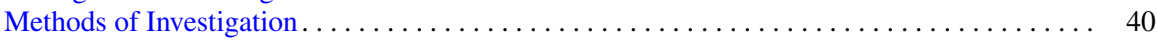

Materials Preparation . .............................. 40

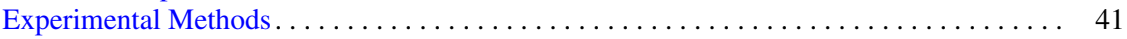

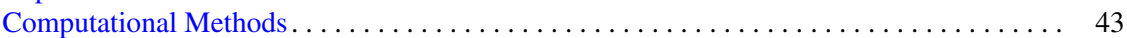

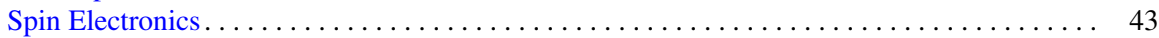

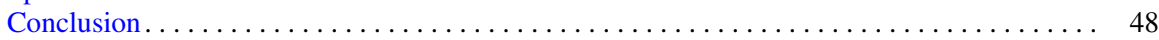

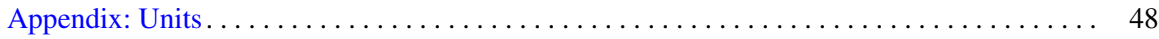

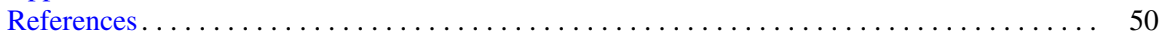

\footnotetext{
J. M. D. Coey $(\bowtie)$

School of Physics, Trinity College, Dublin, Ireland

e-mail: jcoey@tcd.ie
} 


\section{Abstract}

Magnetism is a microcosm of the history of science over more than two millennia. The magnet allows us to manipulate a force field which has catalyzed an understanding of the natural world that launched three revolutions. First came the harnessing of the directional nature of the magnetic force in the compass that led to the exploration of the planet in the fifteenth century. Second was the discovery of the relation between electricity and magnetism that sparked the electromagnetic revolution of the nineteenth century. Third is the big data revolution that is currently redefining human experience while radically transforming social interactions and redistributing knowledge and power.

The emergence of magnetic science demanded imagination and observational acuity, which led to the theory of classical electrodynamics. The magnetic field is associated with electric currents and the angular momentum of charged particles in special materials. Our current understanding of the magnetism of electrons in solids is rooted in quantum mechanics and relativity. Yet only since about 1980 has fundamental theory underpinned rational design of new functional magnetic materials and the conception of new spin electronic devices that can be reproduced on ever smaller scales, leading most notably to the disruptive, 60-year exponential growth of magnetic information storage. The development of new magnetic concepts, coupled with novel materials, device and machine designs has become a rich source of technical innovation.

\section{Introduction}

The attraction of ferrous objects to a permanent magnet has been a source of wonder since the Iron Age. Feeble magnets are widespread in nature in the form of rocks known as lodestones, which are rich in magnetite, an oxide mineral with ideal formula $\mathrm{Fe}_{3} \mathrm{O}_{4}$. Rocky outcrops eventually get magnetized by huge electric currents when lightning strikes, and these natural magnets were known and studied in ancient Greece, Egypt, China, and Mesoamerica. Investigations of magnetic phenomena led to the invention of steel magnets - needles and horseshoes - then electromagnets and eventually the panoply of hard and soft materials that support the modern magnetics industry. Magnetism in a rare example of a science with recorded history goes back well over 2000 years [1, 2].

Theory and practice have been loose partners for most of that time. What people are able to see and rationalize is inevitably conditioned by a priori philosophical beliefs about the world. The scientific method of critically interrogating nature by experimentation and then amassing and exchanging data and ideas among the community of the curious came to be established only gradually. Mathematics emerged as the supporting scaffold of natural philosophy in Europe in the seventeenth century, when precisely formulated natural laws and explanations began to take root. Nevertheless, most of the progress that has been made in magnetism in the past - from the discovery of horseshoe magnets or electromagnetic induction 
to the development of Alnico - was based on intuition and experience, rather than formal theory. That situation is changing.

The discovery of the electron in the closing years of the nineteenth century impelled the great paradigm shift from classical to modern physics. Magnetism, however familiar and practically important it had become, was fundamentally incomprehensible in classical terms. Charged particles were theoretically expected to exhibit no magnetism of any kind. It took 25 years and the insights of quantum mechanics and relativity to resolve that conundrum. Magnetism then went on to play a key role in clarifying basic concepts in condensed matter physics and Earth science over the course of the twentieth century. Now it is a key player in the transformative information technology of the twenty-first century.

\section{Early History}

Aristotle attributed the first reflections on the nature of magnetic attraction to Thales, the early Greek philosopher and mathematician who was born in Miletus in Asia Minor in $624 \mathrm{BC}$. Thales was an animist who credited the magnet with a soul, on account of its ability to create movement, by attraction. This curious idea was to linger until the seventeenth century. The magnet itself is believed to be named after Magnesia, a city in Lydia in Asia Minor that was a good source of lodestone. In the fifth century BC, when Empedokles postulated the existence of the four elements - Earth, water, air, and fire - magnetism was associated with air. Special effluvia somehow passing through the invisible pores in magnetic material were invoked to explain the phenomenon, a theory echoed much later by Descartes in a mechanistic picture that finally laid the magnet's soul to rest. The Roman poet Lucretius writing in the first century BC mentions magnetic induction (the ability of a magnet to induce magnetism in pieces of nonmagnetic iron) and for the first time notes the ability of magnets not just to attract but also to repel one another. The Greek approach of developing a philosophical framework into which natural observations were expected to fit was not conducive to open-minded exploration of the natural world.

\section{The Compass}

The Chinese approach to the magnet was more practical. Their magnetism was initially linked to practical concerns of geomancy and divination [3]. The art of adapting the residences of the living and the tombs of the dead to harmonize with local currents of the cosmic breath demanded knowledge of its direction. A south-pointer consisting of a carved lodestone spoon that was free to rotate on a polished baseplate (Fig. 1) was already in use at the time of Lucretius and may have originated hundreds of years earlier. An important discovery, attributed to Zeng Gongliang in 1064, was that iron could acquire a thermoremanent magnetization when rapidly cooled from red heat in the Earth's magnetic field. A short step 
a

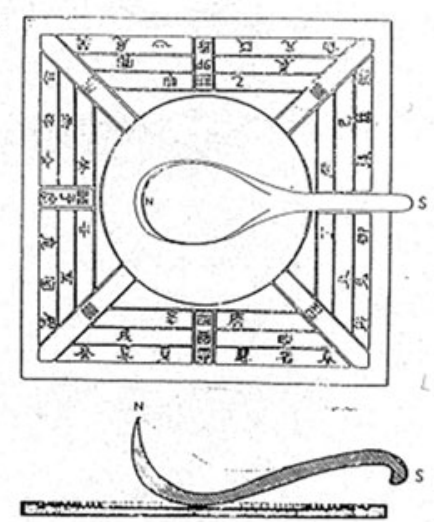

C

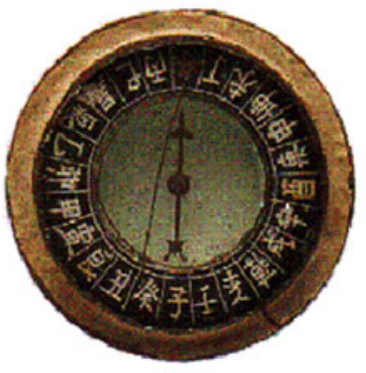

b
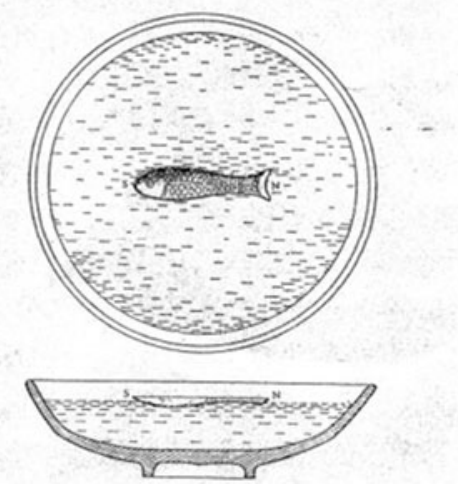

d

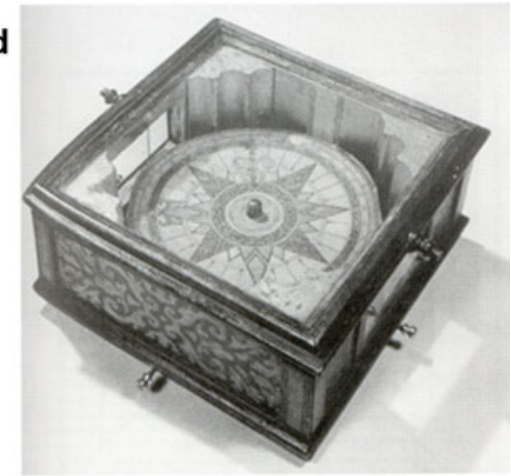

Fig. 1 Magnetic direction finders. (a) Baseplate and lodestone spoon of the south-pointer used in China from about the first century BC (Needham, courtesy of Cambridge University Press). (b) A Chinese floating compass from 1044. (c) Fifteenth-century Chinese and (d) Portuguese mariners' compasses. (Boorstin, courtesy of Editions Robert Laffont)

led to the suspended compass needle, which was described by Shen Kuo around 1088 , together with declination, the deviation of the needle from a north-south axis. Floating compasses had also been developed by this time, often in the form of an iron fish made to float in a bowl of water.

The compass appeared about a century later in Europe, where it was first described by Alexander Neckam in 1190. The direction-finding ability of the magnetic needle or fish was also exploited by Arabs and Persians from the thirteenth century, both for navigation and to determine the sacred direction of Mecca [4]. Compasses (Fig. 1) were the enabling technology for the great voyages of discovery of the fifteenth century, bringing the Ming admiral Cheng Ho to the coasts of Africa in 1433 and Christopher Columbus (who rediscovered declination) to America in 1492, where he landed on the continent where the Olmecs may once have displayed a knowledge of magnetism in their massive stone carvings of human figures and sea turtles dating from the second millennium BC. 
Before long, the landmasses and oceans of our planet were mapped and explored. According to Francis Bacon, writing in Novum Organum in 1620 [5], the magnetic compass was one of three things, along with printing and gunpowder had "changed the whole face and state of things throughout the world." All three were originally Chinese inventions. The compass helped to provide us with an image of the planet we inhabit. This was the first of three occasions when magnetism changed the world.

\section{The Emergence of Modern Science}

A landmark in the history of magnetism in Europe was the work of the French crusader monk Petrus Peregrinus. His tract Epistola de Magnete [6] recounts experiments with floating pieces of lodestone and carved lodestone spheres called terella, which he wrote up in Southern Italy during the 1269 siege of Lucera. He describes how to find the poles of a magnet and relates magnetic attraction to the celestial sphere. The same origin had long been associated with the magnet's directional property in China [3]; we should not forget that before electric light, people were acutely aware of the stars and scrutinized them keenly. Peregrinus's tract included an ingenious proposal for a magnetic perpetual motion device - a theme that has been embraced by charlatans throughout the ages, right up to the present day.

Much credit for the inauguration of the experimental method in a recognizably modern form belongs to William Gilbert. Physician to the English Queen Elizabeth I, Gilbert personally conducted a series of experiments on terellas, which led him to proclaim that the Earth itself was a great magnet. The lodestone or steel magnets aligned themselves not with the celestial sphere, but with the Earth's poles. He induced magnetism by cooling iron in the Earth's field and then destroyed it by heating or hammering. Gilbert was at pains to debunk the millennial accretion of superstition that clung to the magnet, confidently advocating in a robust polemical style reliance on the evidence of one's own eyes. He described his investigations in his masterwork De Magnete, published in 1600 [7]. It is arguably the first modern scientific text.

Subsequent developments were associated with improvements in navigation and the prestige of the great voyages of discovery. Gilbert's theories dominated the seventeenth century up until Edmond Halley's 1692 shell model for the Earth's magnetic structure, which strongly influenced compass technology and navigation. Naval interests were the principal drivers of magnetic research during this period, and Halley was sponsored by the British Navy to survey and prepare charts of the Earth's magnetic field in the North and South Atlantic oceans (Fig. 2), This was in the vain hope of addressing the pressing longitude problem, by pinpointing magnetically the position of a vessel on the Earth's surface.

The following century was marked by the professionalization of natural philosophy (as physical science was then known in Europe) [8]. Accordingly, the natural philosopher with his mantle of theory was rewarded with social status, access to public funding, and credibility beyond that extended to artisans on the one hand and 


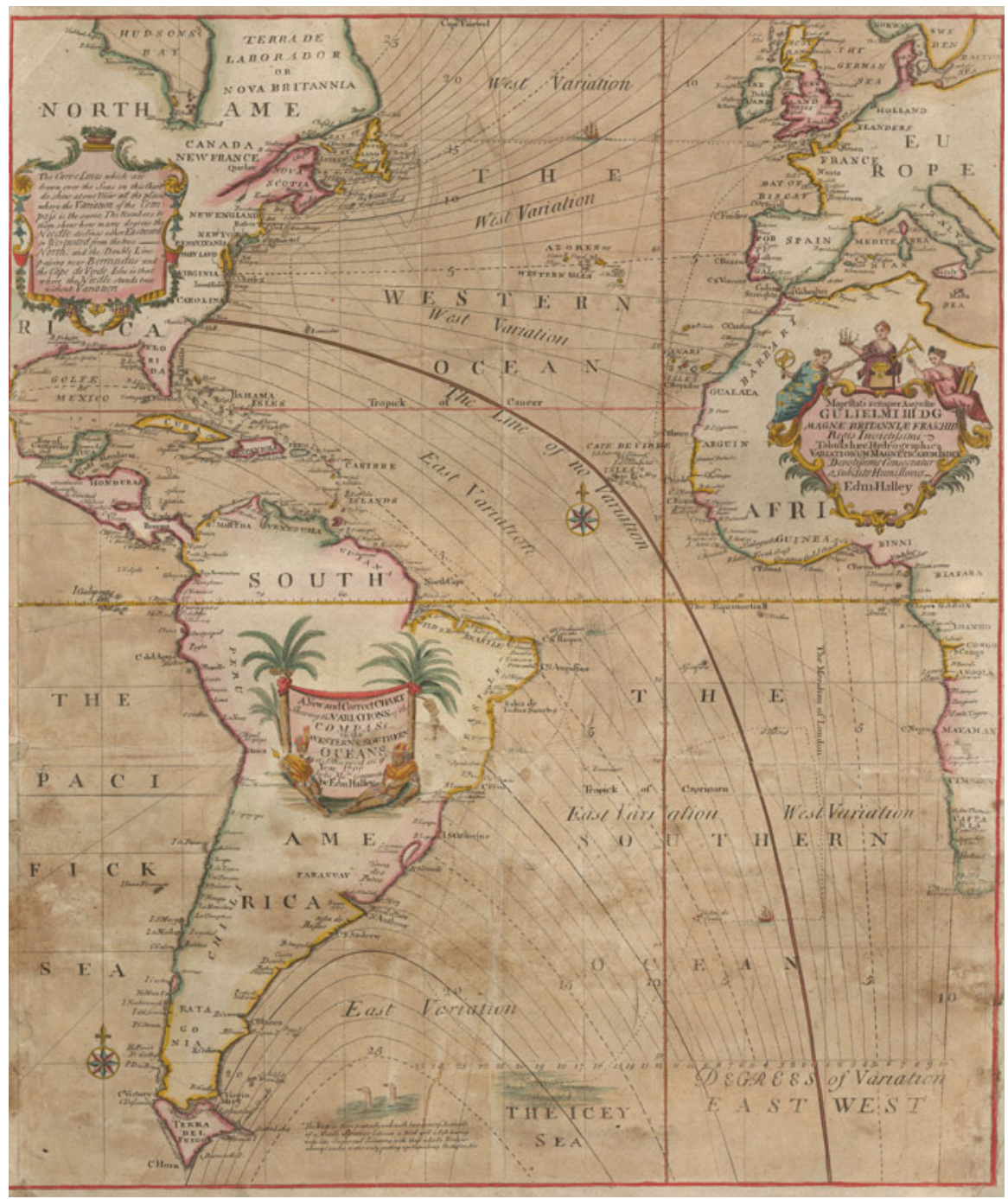

Fig. 2 A section of Halley's world chart of magnetic variation published in 1700

quacks on the other, such as the colorful Anton Mesmer, who propagated theories of animal magnetism in his salon in Paris or James Graham with his royal Patagonian magnetic bed for nightly rental in a fashionable London townhouse. The English entrepreneur Gowin Knight, representative of a new breed of natural philosopher, greatly improved the quality of bar magnets and compasses, coupling scientific endeavor with manufacturing enterprise and a keen sense of intellectual property.

An outstanding technical breakthrough of the eighteenth century was the 1755 discovery by the Swiss blacksmith Johann Dietrich that the horseshoe was an ideal 
shape for a steel magnet [1]. His invention, a clever practical solution to the age-old problem of self-demagnetization in bar magnets, was enthusiastically promoted by his mentor, the Swiss applied mathematician Daniel Bernoulli, who garnered most of the credit.

\section{The Electromagnetic Revolution [9]}

The late eighteenth century in Europe was a time of great public appetite for lectures and demonstration of the latest scientific discoveries, not least in electricity and magnetism. This effervescent age witnessed rapid developments in the harnessing of electricity, with the 1745 invention of the Leyden jar culminating in Alessandro Volta's 1800 invention of the voltaic cell. Analogies between electrostatics and magnetism were tantalizing, but the link between them proved elusive.

\section{Magnetostatics and Classical Electrodynamics}

The torsion balance allowed Charles-Augustin de Coulomb to establish in 1785 the quantitative inverse square laws of attraction and repulsion between electric charges, as well as similar laws between analogous magnetic charge or poles that were supposed to be located near the ends of long magnetized steel needles [2]. The current convention is that the north and south magnetic poles are negatively and positively charged, respectively. His image was of pairs of positive and negative electric and magnetic fluids permeating matter, which became charged if one of them dominated or polarized if they were spatially separated. Unlike their electric counterparts, the magnetic fluids were not free to flow and could never be unbalanced in any piece of magnetic material. Coulomb found that the force $F$ between two magnetic poles separated by a distance $r$ fell away as $1 / r^{2}$. Siméon Denis Poisson then interpreted Coulomb's results in terms of a scalar potential $\varphi_{\mathrm{m}}$, analogous to the one he used for static electricity, such that the magnetic field could be written as $\boldsymbol{H}(\boldsymbol{r})=-\nabla \varphi_{\mathrm{m}}$. In modern terms, $\varphi_{\mathrm{m}}$ is measured in amperes, and $\boldsymbol{H}$ in $\mathrm{Am}^{-1}$. Magnetic charge $q_{\mathrm{m}}$ is measured in Am, and the corresponding potential $\varphi_{\mathrm{m}}=q_{\mathrm{m}} / 4 \pi r$. The magnetic field due to a charge is $\boldsymbol{H}(\boldsymbol{r})=\mathrm{q}_{\mathrm{m}} \boldsymbol{r} / 4 \pi r^{3}$, and Coulomb's inverse square law for the force between two charges separated by $\boldsymbol{r}$ is $\boldsymbol{F}=\mu_{0} q_{\mathrm{m}} q_{\mathrm{m}}{ }^{\prime} \boldsymbol{r} / 4 \pi r^{3}$. Here $\mu_{0}$ is the magnetic constant, $4 \pi 10^{-7} \mathrm{NA}^{-2}$, which appears whenever the magnetic field $\boldsymbol{H}$ interacts with matter. (Other equivalent ways of writing the units of $\mu_{0}$ are $\mathrm{Hm}^{-1}$ or $\mathrm{TmA}^{-1}$.)

In Poisson's opinion, the practice and teaching of mathematics were the purpose of life. He developed his mathematical theory of magnetostatics from 1824, which included the equation that bears his name $\nabla^{2} \varphi_{\mathrm{m}}=-\rho_{\mathrm{m}}$, where $\rho_{\mathrm{m}}$ is the density of magnetic poles. However, the association of $\boldsymbol{H}$ with a scalar potential is only valid in a steady state and when no electric currents are present. The coulombian picture of the origin of magnetic fields was dominant in textbooks until about 1960, and it persists in popular imagery. 
A revolutionary breakthrough in the history of magnetism came on 21st April 1820 , with the discovery of the long-sought link between electricity and magnetism. During a public lecture, the Danish scientist Hans Christian Oersted noticed that a compass needle was deflected as he switched on an electric current in a copper wire. His report, published in Latin a few months later, triggered an experimental frenzy. As soon as the news reached Paris, François Arago (who briefly served as President of France in 1848) immediately performed an experiment that established that a current-carrying conducting coil behaved like a magnet. A week after Arago's report, André-Marie Ampère presented a paper to the French Academy suggesting that ferromagnetism in a magnetized body was caused by internal currents flowing perpendicular to the axis of magnetization and that it should therefore be possible to magnetize steel needles in a solenoid. Together with Arago, he successfully demonstrated his ideas in November 1820, showing that current loops and coils were functionally equivalent to magnets, and he subsequently established the law of attraction or repulsion between current-carrying wires. Ten days later, the British scientist Humphrey Davy had similar results. The electromagnet was invented by William Sturgeon in 1825; within 5 years Joseph Henry had used a powerful electromagnet in the USA for the first electric telegraph. As early as 1822, Davy's assistant Michael Faraday produced the first rudimentary electric motor, and Ampère envisaged the possibility that the currents causing magnetism in solids were "molecular" rather than macroscopic in nature.

In formal terms, Ampère's equivalence between a magnet and a current loop of area $A$ carrying a current $I$ is expressed as

$$
m=I A
$$

where $A$ is in square meters, $I$ is in amperes, and the magnetic moment $m$ is therefore in $\mathrm{Am}^{2}$. Magnetization, defined in a mesoscopic volume $V$ as $\boldsymbol{M}=m / V$, has units $\mathrm{Am}^{-1}$. The direction of $m$ is conventionally related to that of the electric current by the right-hand rule. At the same time as the experimental work of Ampère and Arago, Jean-Baptiste Biot and Félix Savart formulated the law expressing the relation between a current and the field it produces. A current element $I \delta \boldsymbol{l}$ generates a field $\delta \boldsymbol{H}=I \delta \boldsymbol{l} \times \boldsymbol{r} / 4 \pi \boldsymbol{r}^{3}$ at a distance $\boldsymbol{r}$. Integrating around a current loop yields an expression for the $H$-field due to the moment $\boldsymbol{m}$ :

$$
\boldsymbol{H}=[3(\boldsymbol{m} . \boldsymbol{r}) \boldsymbol{r}-\boldsymbol{m}] / 4 r^{3}
$$

The form of the field represented by Eq. (2) and illustrated in Fig. 3 is identical to that of an electric dipole, so $m$ is often referred to as a magnetic dipole although we have no evidence for the existence of independent magnetic poles. The dipole moment is best represented by an arrow in the direction of $\boldsymbol{m}$, although it is still commonplace to see the north-seeking and south-seeking poles of a magnet denoted by the letters $\mathrm{N}$ and $\mathrm{S}$. Old habits die hard.

Magnetic moments tend to align with magnetic fields in which they are placed. The torque on the dipole $m$ is $\boldsymbol{\Gamma}=\mu_{0} \boldsymbol{m} \times \boldsymbol{H}$, and the corresponding energy of the 


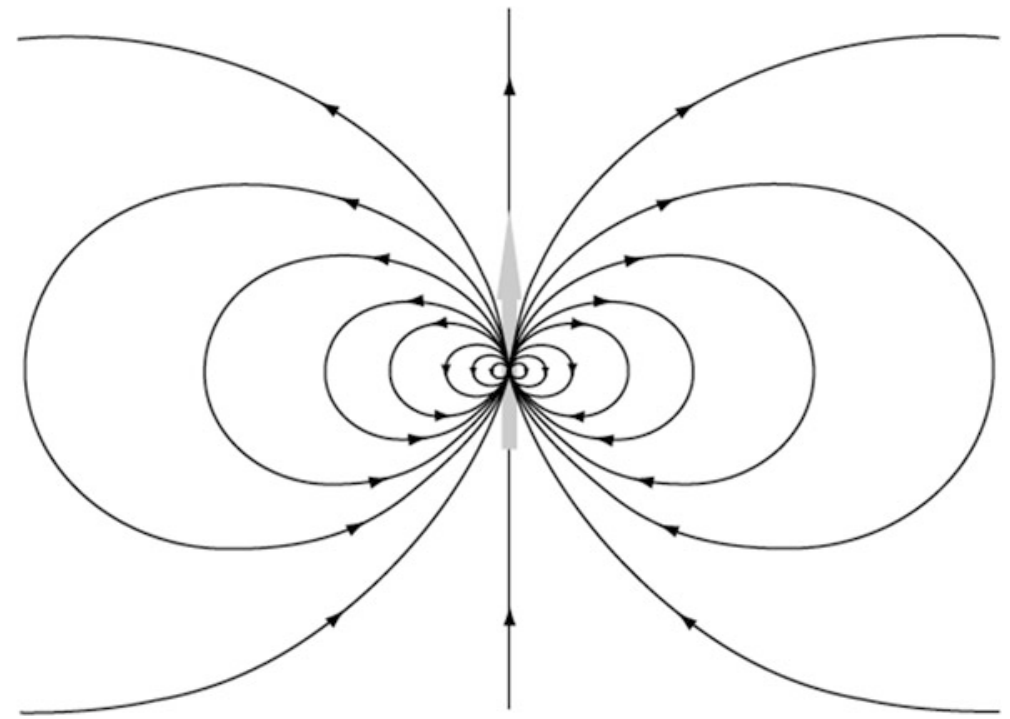

Fig. 3 Contours of equal magnetic field produced by a magnetic dipole moment $m$, represented by the grey arrow

dipole is $E=-\mu_{0} \boldsymbol{m} . \boldsymbol{H}$. These equations are better written in terms of the more fundamental magnetic field $\boldsymbol{B}$, as discussed below; in free space the two are simply proportional, $\boldsymbol{B}=\mu_{0} \boldsymbol{H}$, so the torque is

$$
\Gamma=m \times B
$$

and the corresponding energy is

$$
E=-\boldsymbol{m} \cdot \boldsymbol{B}
$$

The two rival descriptions of magnetization in solids following from the work of Coulomb or Ampère, based either on magnetic poles or on electric currents, have colored thinking about magnetism ever since (Fig. 4). The poles have no precise, independent physical reality; they are fictitious entities that are a mathematicallyconvenient way to represent the $H$-field, which is of critical importance in magnetism because it is the local $H$-field that determines the state of magnetization of a solid. Currents are closer to reality; electric current loops exist, and they do act like magnets. Although it is difficult to attribute the intrinsic spin moment of the electron to a current, the amperian picture of the origin of magnetic fields is generally adopted in modern textbooks.

Nineteenth-century electromagnetism owed much to the genius of Michael Faraday. Guided entirely by observation and experiment, with no dependence on formal theory, he was able to perfect the concept of magnetic field, which he 
Fig. 4 Alternative coulombian (left) and amperian (right) descriptions of the magnetization of a uniformly magnetized cylinder, with a magnetic dipole moment $\boldsymbol{m}$ in the direction represented by the black arrow; $\sigma_{\mathrm{m}}{ }^{ \pm}$is the surface magnetic charge density, $\boldsymbol{j}_{\mathrm{ms}}$ is the surface electric current density
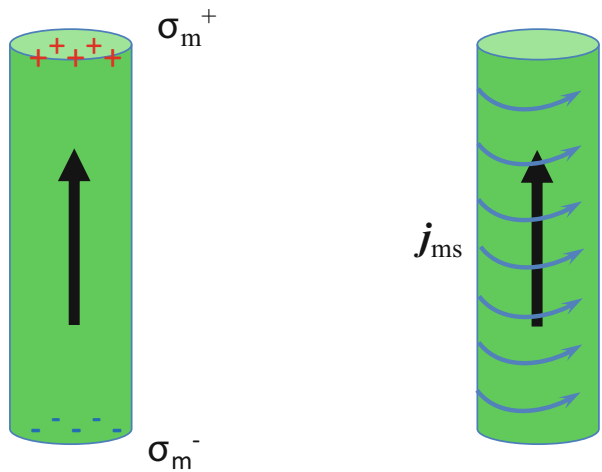

described by lines of force [10]. Faraday classified substances in three magnetic categories. Ferromagnets like iron were spontaneously magnetized and strongly attracted into a magnetic field; paramagnets were weakly magnetized by a field and feebly drawn into the regions where the field was strongest; diamagnets, on the contrary, were weakly magnetized opposite to the field and repelled by it. Working with an electromagnet, he discovered the law that bears his name and the phenomenon of electromagnetic induction - that a flow of electricity can be induced by a changing magnetic field - in 1831. His conviction that a magnetic field should have some effect on light led to his 1845 discovery of the magneto-optic Faraday effect - that the plane of polarization of light rotates upon passing through a transparent medium in a direction parallel to the magnetization of the medium.

The epitome of classical electrodynamics was the set of equations formulated in 1865 by James Clerk Maxwell, the Scottish theoretician, who had "resolved to read no mathematics on the subject till he had first read through Faraday's 'Experimental Researches in Electricity'." Maxwell's magnificent equations formally defined the relationship between electricity, magnetism, and light [11]. As reformulated by Oliver Heaviside, the equations are a succinct statement of classical electrodynamics. In the opinion of Richard Feynman, Maxwell's discovery of the laws of electrodynamics was the most significant event of the nineteenth century. The equations in free space are formulated in terms of the fundamental magnetic and electric fields $\boldsymbol{B}$ and $\boldsymbol{E}$. Using the international system of SI units adopted in this Handbook, the equations read:

$$
\begin{aligned}
\nabla . \boldsymbol{B} & =0 \\
\varepsilon_{0} \nabla . \boldsymbol{E} & =\rho \\
\left(1 / \mu_{0}\right) \nabla \times \boldsymbol{B} & =\boldsymbol{j}+\varepsilon_{0} \partial \boldsymbol{E} / \partial t \\
\nabla \times \boldsymbol{E} & =-\partial \boldsymbol{B} / \partial t
\end{aligned}
$$

The first and third equations express the idea that there are no sources of the magnetic B-field other than time-varying electric fields and electric currents of 
density $\boldsymbol{j}$, whereas the second and fourth equations show that the electric field results from electric charge density $\rho$ and time-varying magnetic fields. Maxwell's equations are invariant in a moving frame of reference, although the relative magnitudes of $\boldsymbol{E}$ and $\boldsymbol{B}$ are altered.

The famous wavelike solutions of these equations in the absence of charges and currents are electromagnetic waves, which propagate in free space with velocity $c=1 /\left(\varepsilon_{0} \mu_{0}\right)^{1 / 2}$. In SI, the definition of the magnetic constant $\mu_{0}$ is linked to the fine structure constant. To nine significant figures, it is equal to $4 \pi 10^{-7} \mathrm{NA}^{-2} \cdot \varepsilon_{0}$ is then related to the definition of the velocity of light. Heinrich Hertz demonstrated Maxwell's electromagnetic waves experimentally in 1888, and he showed that their behavior was essentially the same as that of light. Hertz could think of no practical application for his work, yet within a few decades, it had become the basis of radio broadcasting and wireless communication!

The mechanical effects of electric and magnetic fields were summarized by Hendrik Lorentz in his expression for the force density $\boldsymbol{F}_{\mathrm{L}}$ :

$$
\boldsymbol{F}_{\mathrm{L}}=\rho \boldsymbol{E}+\boldsymbol{j} \times \boldsymbol{B}
$$

The equivalent expression for the force on a particle of charge $q$ moving with velocity $v$ is $\boldsymbol{f}=q(\boldsymbol{E}+\boldsymbol{v} \times \boldsymbol{B})$.

Two further fields $\boldsymbol{H}$ and $\boldsymbol{D}$ are introduced in the formulation of Maxwell's equations in a material medium to circumvent the inaccessibility of the current and charge distributions in the medium. We have no direct way of measuring the atomic charges associated with the polarization of a ferroelectric material or the atomic currents associated with the magnetization of a ferromagnetic material, so we define $\boldsymbol{H}$ and $\boldsymbol{D}$ in terms of fields created by the measurable free charges $\rho$ and free currents $\boldsymbol{j}$, with dipolar contributions from the magnetization $\boldsymbol{M}$ or polarization $\boldsymbol{P}$ of any magnetic or dielectric material that may be present. The equations now read:

$$
\begin{aligned}
\nabla . \boldsymbol{B} & =0 \\
\nabla . \boldsymbol{D} & =\rho \\
\nabla \times \boldsymbol{H} & =\boldsymbol{j}+\partial \boldsymbol{D} / \partial t \\
\nabla \times \boldsymbol{E} & =-\partial \boldsymbol{B} / \partial t
\end{aligned}
$$

They are further simplified in a static situation when the time derivatives are zero. The new fields are trivially related to $\boldsymbol{B}$ and $\boldsymbol{E}$ in free space since $\boldsymbol{B}=\mu_{0} \boldsymbol{H}$ and $\boldsymbol{D}=\varepsilon_{0} \boldsymbol{E}$, but in a material medium, the $\boldsymbol{H}$-field is defined in terms of the $\boldsymbol{B}$-field and the magnetization $\boldsymbol{M}$ (the magnetic moment per unit volume) as $\boldsymbol{H}=\boldsymbol{B} / \mu_{0}-\boldsymbol{M}$ or

$$
\boldsymbol{B}=\mu_{0}(\boldsymbol{H}+\boldsymbol{M})
$$



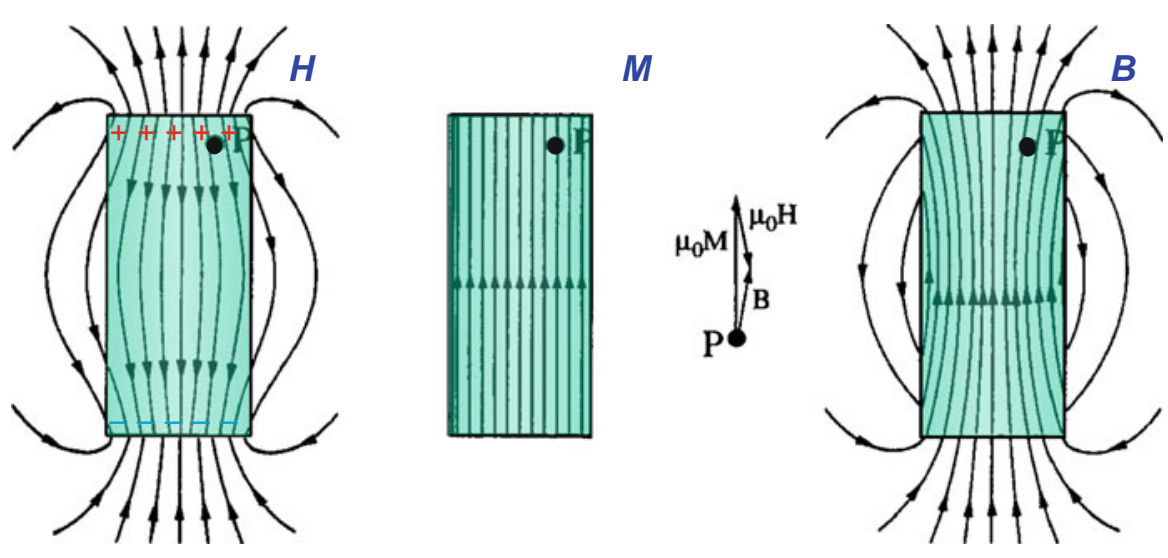

$$
\boldsymbol{B}=\mu_{0}(\boldsymbol{H}+\boldsymbol{M})
$$

Fig. $5 \boldsymbol{B}, \boldsymbol{H}$, and $\boldsymbol{M}$ for a uniformly magnetized ferromagnetic bar. Eq. (8) is represented by the vector triangle. The $H$-field can be regarded as originating from a distribution of positive and negative magnetic charge (south and north magnetic poles) on opposite faces

Likewise $\boldsymbol{D}=\varepsilon_{0}(\boldsymbol{E}+\boldsymbol{P})$, where $\boldsymbol{P}$ is the electric polarization. To specify a situation in magnetostatics or electrostatics, any two of the three magnetic or electric fields are needed. (Magnetization $\boldsymbol{M}$ and polarization $\boldsymbol{P}$ are regarded as vector fields.) The defining relation between $\boldsymbol{B}, \boldsymbol{H}$, and $\boldsymbol{M}$ for a uniformly magnetized ferromagnetic bar is illustrated in Fig. 5. Note that the $B$-field is solenoidal - the field lines are continuous with no sources or sinks; it is divergenceless and can therefore be expressed as the curl of a vector potential $\boldsymbol{A}$ - whereas the $H$-field is conservative; it is irrotational provided $\boldsymbol{j}$ is zero and can be expressed as the gradient of a scalar potential. Outside the magnet, the $H$-field is called the stray field, but within the magnet where it is oppositely oriented to $\boldsymbol{M}$, the name changes to demagnetizing field. Boundary conditions that $B_{\perp}$ and $H_{\|}$are continuous across an interface in a steady state $(\boldsymbol{j}=0)$ follow from the first and third of Maxwell's equations 7. $\boldsymbol{B}$ is the fundamental magnetic field, because no elementary magnetic poles exist in nature $(\nabla \cdot \boldsymbol{B}=0)$, but it is the local value of $\boldsymbol{H}$ (and perhaps the sample history) that determines the magnetic state of a solid, including its micromagnetic domain structure. The $H$-field acting in a solid is the sum of the applied field $\boldsymbol{H}^{\prime}$ and the local demagnetizing field $\boldsymbol{H}_{\mathrm{d}}$ created by the solid body itself.

When describing the stray field outside a distribution of magnetization $\boldsymbol{M}(\mathbf{r})$ in a solid, the coulombian and amperian descriptions are formally equivalent. The coulombian expression for the magnetic field is obtained by integrating the expression for the field due to a distribution of a magnetic charge $q_{\mathrm{m}}$ per unit volume $\rho_{\mathrm{m}}=-\nabla \cdot \boldsymbol{M}$ in the bulk, and per unit area $\sigma_{\mathrm{m}}=\boldsymbol{M} . \mathbf{e}_{\mathbf{n}}$ at the surface, where $\mathbf{e}_{\mathbf{n}}$ is the unit vector normal to the surface: 


$$
\boldsymbol{H}(\boldsymbol{r})=\frac{1}{4 \pi}\left\{-\int_{V} \frac{\left(\nabla^{\prime} \cdot \boldsymbol{M}\right)\left(\boldsymbol{r}-\boldsymbol{r}^{\prime}\right)}{\left|\boldsymbol{r}-\boldsymbol{r}^{\prime}\right|^{3}} \mathrm{~d}^{3} r^{\prime}+\int_{S} \frac{\boldsymbol{M} \cdot \boldsymbol{e}_{n}\left(\boldsymbol{r}-\boldsymbol{r}^{\prime}\right)}{\left|\boldsymbol{r}-\boldsymbol{r}^{\prime}\right|^{3}} \mathrm{~d}^{2} r^{\prime}\right\}
$$

This formula gives $\boldsymbol{H}(\boldsymbol{r})$ both inside and outside the magnetic material. Outside $\boldsymbol{B}(\boldsymbol{r})=\mu_{0} \boldsymbol{H}(\boldsymbol{r})$.

The amperian expression for the magnetic field produced by a distribution of currents is based on the Biot-Savart expression for the field due to a current element, including contributions from the current density $\boldsymbol{j}_{\mathbf{m}}=\nabla \times \boldsymbol{M}$ in the bulk, and $j_{\mathbf{m s}}=\boldsymbol{M} \times \mathbf{e}_{\mathrm{n}}$ at the surface:

$$
\boldsymbol{B}(\boldsymbol{r})=\frac{\mu_{0}}{4 \pi}\left\{\int_{\mathrm{V}} \frac{\left(\nabla^{\prime} \times \boldsymbol{M}\right) \times\left(\boldsymbol{r}-\boldsymbol{r}^{\prime}\right)}{\left|\boldsymbol{r}-\boldsymbol{r}^{\prime}\right|^{3}} \mathrm{~d}^{3} r^{\prime}+\int_{\mathrm{S}} \frac{\left(\boldsymbol{M} \times \boldsymbol{e}_{n}\right) \times\left(\boldsymbol{r}-\boldsymbol{r}^{\prime}\right)}{\left|\boldsymbol{r}-\boldsymbol{r}^{\prime}\right|^{3}} \mathrm{~d}^{2} r^{\prime}\right\}
$$

This formula gives $\boldsymbol{B}(\boldsymbol{r})$ both inside and outside the magnetic material. The same result can be obtained by appropriate integration of Eq. 2 over a magnetization distribution $\boldsymbol{M}(\boldsymbol{r})$ [12].

For uniformly magnetized ellipsoids, the demagnetizing field $\boldsymbol{H}_{\mathrm{d}}$ is related to the magnetization by

$$
\boldsymbol{H}_{\mathrm{d}}=-\mathcal{N} \boldsymbol{M}
$$

where $\mathcal{N}$ is a tensor with unit trace [13]. It reduces to a simple scalar demagnetizing factor $0<\mathcal{N}<1$ when the magnetization lies along a principal axis of the ellipsoid. $\mathcal{N} \approx 0$ for a long needle magnetized along its axis, and $\mathcal{N}=1$ for a flat plate magnetized perpendicular to the plane. A sphere has $\mathcal{N}=1 / 3$. For any shape less symmetric than an ellipsoid, the demagnetizing field is nonuniform. There are useful approximate formulae for square bars and cylinders [14], such as $1 /(2 n+1)$ and $1 /[(4 n / \sqrt{ } \pi)+1]$, respectively, but they should not obscure the fact that the demagnetizing field in these shapes really is quite nonuniform. Here $n$ is the ratio of length to diameter. The demagnetizing field is the reason why for centuries magnets were condemned to take awkward shapes of bars or horseshoes to avoid substantial self-demagnetization and why the most successful electromagnetic machines of the nineteenth century were built around electromagnets rather than permanent magnets. The hardened steel magnets of the day showed little coercivity and were easily demagnetized. Demagnetizing fields are also the cause of ferromagnetic domains. The shape constraint on permanent magnets was not lifted until the middle of the twentieth century. Permanent magnets then came to the fore in the design of electric motors and magnetic devices. Fig. 6 illustrates a collection of magnets from the eighteenth, nineteenth, and twentieth centuries.

The imaginative world of Maxwell and his followers in the latter part of the nineteenth century when the electromagnetic revolution was in full swing was 


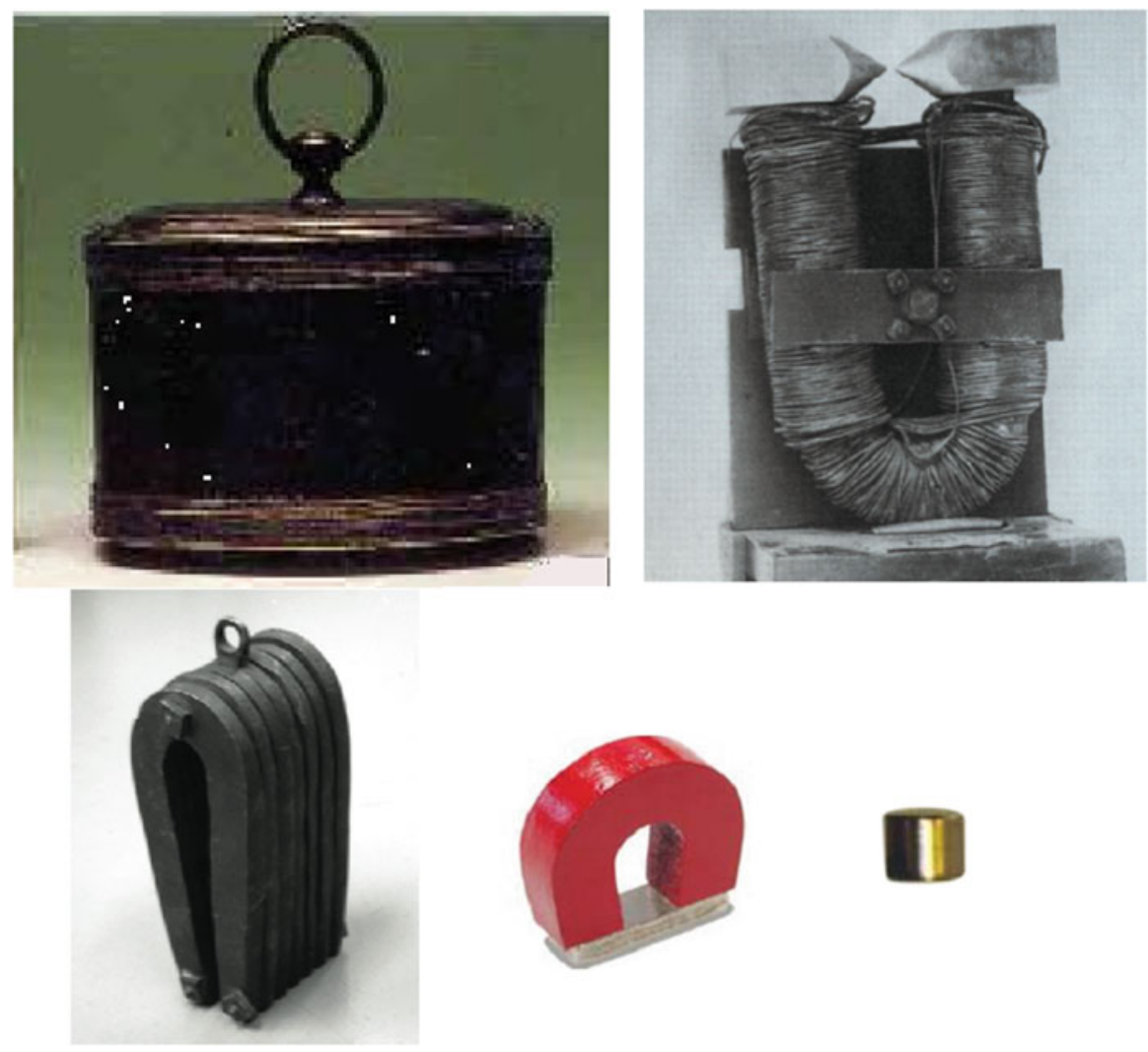

Fig. 6 Magnets from four centuries; top, seventeenth-century lodestone, nineteenth-century electromagnet; bottom, eighteenth-century horseshoe magnet, twentieth-century alnico and $\mathrm{Nd}_{2} \mathrm{Fe}_{14} \mathrm{~B}$ magnets (not to scale)

actually far removed from our own [15]. They envisaged light and other Hertzian waves as propagating in an all-pervasive aether, which was believed to possess magical mechanical properties - it had to be a massless incompressible fluid, transparent and devoid of viscosity, yet millions of times more rigid than steel! Elaborate mechanical models were envisaged for the waves and fields. In due course it came to be understood that reality was represented by the abstract mathematics, which remained after all the mechanical props had been discarded.

\section{The Earth's Magnetic Field}

The Earth's field was the prime focus of attention of magnetism for over a millennium, especially after it was understood that the magnetic field was of terrestrial origin. By the beginning of the nineteenth century, the components of the field were 
being recorded regularly in laboratories across the world. A comparison of the daily magnetic records at Paris and Kazan, cities lying $4000 \mathrm{~km}$ apart, for the same day in 1825, showed astonishingly similar short-term fluctuations. This inspired Carl Friedrich Gauss to establish a worldwide network of 50 magnetic observatories, coordinated from Göttingen, to make meticulous simultaneous measurements of the Earth's field, in the hope that if enough high-quality data could be collected, the mystery of its origin and its fluctuations might be solved. This heroic pioneering venture in international scientific collaboration amassed stores of data that were enormous for that time. It inspired Gauss to develop spherical harmonic analysis, from which he calculated that the leading, dipolar term accounted for about $90 \%$ of the field and that the origin of the stable component was essentially internal. Edward Sabine later spotted that the intensity of the short-term fluctuations tracked the 11-year sunspot cycle, which we now know corresponds to reversals of the solar magnetic field. But in its primary aim, Gauss's Magnetische Verein must be counted a failure. No amount of data, however copious and precise, could reveal a deterministic origin of a phenomenon that was fundamentally chaotic. Piles of data with no theory or hypothesis through which to view and be tested by them are not very informative. This lesson was learned slowly.

The pole picture of the Earth's magnetic field, albeit with poles that needed to travel tens of kilometers every year to account for the secular variation, yielded eventually in the academy if not in the popular imagination to one based on electric currents driven by convection in the Earth's liquid core. Joseph Larmor, a dogged believer in the aether, was an early proponent of the geomagnetic dynamo. He demonstrated the precession of a magnet in a magnetic field at a frequency $f_{L}=\gamma B / 2 \pi$ that bears his name. The precession is analogous to that of a spinning top in a gravitational field; it is a consequence of the torque on a magnetic moment expressed by Eq. 3. The constant $\gamma$, known as the gyromagnetic ratio, is the ratio of the magnetic moment to its associated angular momentum. The proportionality of these two quantities that at first sight appear quite dissimilar, the famous Einstein-de Haas effect, was eventually demonstrated experimentally in 1915 (Fig. 7).

Fig. 7 The Einstein-de Haas experiment. The iron rod suspended from a torsion fiber twists when a magnetizing current in the surrounding solenoid is reversed, thereby demonstrating the relationship between magnetism and angular momentum

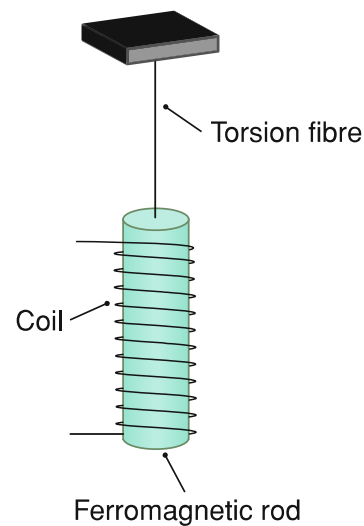




\section{The Properties of Ferromagnets}

If the luminiferous aether was inaccessible to experimental investigation, as the 1887 Michelson-Morley experiment suggested, the same could not be said for magnetic materials. With its focus on electromagnetism, the nineteenth century brought a flurry of investigations of the magnetic properties of the ferromagnetic metals, iron (discovered in the fourth millennium BC), cobalt (discovered in 1735), and nickel (discovered in 1824) and some of their alloys, which were at the heart of electromagnetic machines. In 1842 James Joule, a brewer and natural philosopher, discovered the elongation of an iron bar when it was magnetized to saturation and demonstrated in a liquid displacement experiment that the net volume was unchanged in the magnetostrictive process, owing to a compensating contraction in the perpendicular directions [16]. Magnetostriction is the reason why transformers hum. Gustav Wiedemann observed that an iron bar twisted slightly when a current was passed through it in the presence of a magnetic field. Anisotropic magnetoresistance (AMR) was discovered by William Thomson in 1856; the resistance of iron or nickel is a few percent higher when measured in the direction parallel to the magnetization than in the perpendicular direction [17]. The Hall effect, the appearance of a transverse voltage when a current was passed through a gold foil subject to a transverse magnetic field was discovered by Edwin Hall in 1879 , And the contribution e proportioal to the magnetization of a ferromagnet tha anomalous Hall effect — was found shortly afterwards, in iron. John Kerr showed in 1877 that the rotation of the plane of polarization of electromagnetic radiation, demonstrated by Faraday for light passing through glass, could also be measured in reflection from polished ferromagnetic metal surfaces [18].

Gauss's collaborator Wilhelm Weber, who had constructed the first electromagnetic telegraph in 1833 , formally presented the idea that molecules of iron were capable of movement around their centers, suggesting that they lay in different directions in an unmagnetized material, but aligned in the same direction in the presence of an applied magnetic field. This was the origin of the explanation of hysteresis by James Alfred Ewing, who coined the name for the central phenomenon of ferromagnetism that he illustrated using a board of small, pivoting magnets [19]. Ewing's activities as a youthful scottish professor at the University of Tokyo in the 1890s helped to establish the strong Japanese school of research on magnetic materials that thrives to the present day.

The hysteresis loop, illustrated in Fig. 8, is the icon of ferromagnetism. Except in very small particles, a magnetized state is always metastable. The saturated magnetic state is higher in energy relative to a multidomain state on account of the demagnetizing field that creates a positive magnetostatic self-energy $-1 / 2 \mu_{0} \int \boldsymbol{M}_{\mathrm{s}} \cdot \boldsymbol{H}_{\mathrm{d}}$ $\mathrm{d} V$ in the fully magnetized state, where the only contribution to the integral comes from the magnet volume. The hardened steel magnets of the nineteenth century showed little coercivity, $H_{\mathrm{c}} \ll M_{\mathrm{s}}$, and could only survive as bars and horseshoes where the demagnetizing factor $\mathcal{N}$ of Eq. 11 was $\ll 1$. The principal achievement in technical magnetism in the twentieth century was the mastery of coercivity; this needed new materials having $H_{\mathrm{c}} \gtrsim M_{\mathrm{s}}$. 


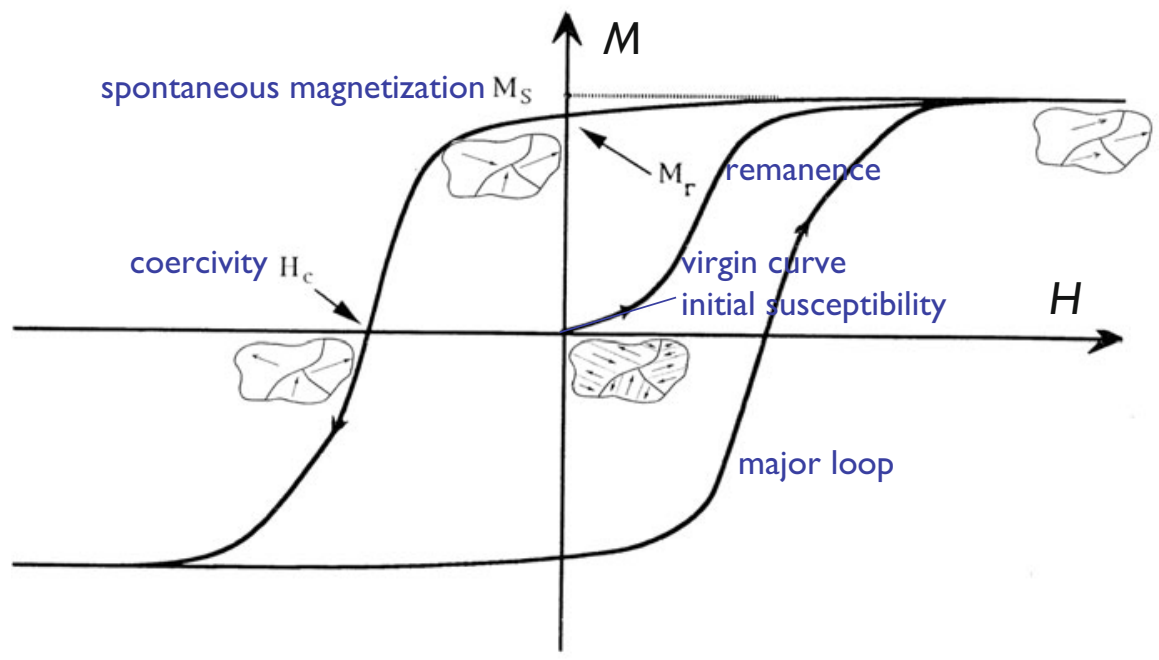

Fig. 8 The hysteresis loop of magnetization $\boldsymbol{M}$ against magnetic field $\boldsymbol{H}$ for a typical permanent magnet, showing the initial magnetization curve from the equilibrium multidomain state and the major loop. $M_{s}$ is the saturation magnetization, $M_{r}$ the remanent magnetization at zero field, and $H_{c}$ the coercive field required to reduce the magnetization to zero

The astonishing transformation of science and society that began in 1820 deserves the name electromagnetic revolution. By the end of the century, electromagnetic engineering was electrifying the planet, changing fundamentally our communications and the conditions of human life and leisure. Huge electric generators, powered by hydro or fossil fuel, connected to complex distribution networks were bringing electric power to masses of homes and factories across the Earth. Electric light banished the tyranny of night. Electric motors of all sorts were becoming commonplace, and public transport was transformed. Telegraph and telephone communication connected people across cities, countries, and continents. Valdemar Poulsen demonstrated magnetic voice recording in 1898. Much of the progress was achieved by engineers who relied on practical knowledge of electrical circuits and magnetic materials, independently of the conceptual framework of electrodynamics that had been developed by the physicists.

The electromagnetic revolution and the subsequent electrification of the planet were the second occasion when magnetism changed the world. The century closed with Pierre Curie's 1895 accurate measurements of the Curie point $T_{\mathrm{C}}$ (the critical temperature above which a material abruptly loses its ferromagnetism) and with the all-important discovery of the electron. Yet ferromagnetism was hardly understood at all at a fundamental level at the turn of the century, and it was becoming evident that classical physics was not up to the task. 


\section{Magnetism of the Electron}

The discovery of the electron in the closing years of the nineteenth century was a huge step toward the modern understanding of magnetism. The elementary charged particle with mass $m_{e}=9.10910^{-31} \mathrm{~kg}$ and charge $e=-1.60210^{-19} \mathrm{C}$ had been named by the Irish scientist George Johnstone Stoney in 1891, several years before Jean Perrin in France actually identified negatively charged particles in a cathode ray tube and J. J. Thompson in England measured their charge to mass ratio $\mathrm{e} / \mathrm{m}_{e}$, by deflecting the electrons in a magnetic field and making use of Eq. 6. Another Irish scientist, George Francis FitzGerald, suggested in 1900 that magnetism might be due to rotational motion of these electrons. They turned out to be not only the carriers of electric current but also the essential magnetic constituent of atoms and solids.

\section{The Demise of Classical Physics}

At the beginning of the twentieth century, the contradictions inherent in contemporary physics could no longer be ignored, but 25 years were to elapse before they could be resolved. In that heroic period, classical physics and the lingering wisps of aether were blown away, and a new paradigm was established, based on the principles of quantum mechanics and relativity.

Magnetism in particular posed some serious puzzles. In order to account for the abrupt disappearance of ferromagnetism at the Curie point, Pierre Weiss, who had developed Ewing's concept of magnetic domains, postulated in 1907 the existence of an internal molecular field.

$$
\boldsymbol{H}_{i}=n_{\mathrm{W}} \boldsymbol{M}
$$

proportional to magnetization in order to explain the spontaneous magnetization within them. His theory of ferromagnetism was based on Paul Langevin's 1905 explanation of the Curie law susceptibility of an array of disordered classical magnetic moments.

$$
\chi=C / T
$$

Susceptibility $\chi$ can be conveniently defined as the dimensionless ratio $M / H$, where $H$ is the applied magnetic field. The expression is modified for a ferromagnet above its Curie point where it becomes the Curie-Weiss law $\chi=C /\left(T-\theta_{\mathrm{p}}\right)$ with $\theta_{\mathrm{p}} \approx T_{\mathrm{C}}$. With Eq. (12) and Langevin's theory of paramagnetism, Weiss invented the first mean-field theory of a phase transition. For iron, where $M=1.71 \mathrm{MAm}^{-1}$, the Weiss constant $n_{\mathrm{W}}$ is roughly 1000 . According to Maxwell's equation $\nabla . \boldsymbol{B}=0$, the component of $B$ normal to the surface of a magnet is continuous, so there should 
be a stray field of order $\mu_{0} H_{\mathrm{s}} \sim 1000 \mathrm{~T}$ in the vicinity of a magnetized iron bar. In fact, the observed stray fields are a thousand times smaller.

Furthermore if, as Ampère believed, all magnetism was traceable to circulating electric currents, the magnetization of an iron bar requires an incredible surface current of 17,100 A for every centimeter of its length. How could such a current be sustained indefinitely? Why does the iron not melt? What did the sobriquet molecular really mean? The anomalous Zeeman splitting of spectral lines in a magnetic field was another mystery. In retrospect, the most startling result was a theorem proved independently in their theses by Niels Bohr in 1911 and Hendrika van Leeuwen in 1919. They showed that at any finite temperature and in any magnetic or electric field, the net magnetization of a collection of classical electrons vanishes identically. So, in stark contrast with experiment, classical electron physics was fundamentally incompatible with any kind of magnetism!

By 1930, quantum mechanics and relativity had ridden to the rescue, and a new understanding of magnetism emerged in terms of the physics of Einstein, Bohr, Pauli, Dirac, Schrödinger, and Heisenberg. The source of magnetism in matter was identified with the angular momentum of elementary particles, especially the electron [20]. The connection between angular momentum and magnetism had been demonstrated directly on a macroscopic scale in 1915 by the Einsteinde Haas experiment (Fig. 7), where angular recoil of a suspended iron rod was observed when its magnetization was reversed by an applied field. It turned out that the perpetual currents in atoms were quantized in stationary states that did not decay and that the angular momentum of the orbiting electrons was a multiple of Planck's constant $\hbar=1.05510^{-34}$ Js. Furthermore, the electron itself possessed an intrinsic angular momentum or $\operatorname{spin}[20]$ with eigenvalues of $\pm 1 / 2 \hbar$ along the axis of quantization defined by an external field. Weiss's molecular field was no magnetic field at all, but a manifestation of electrostatic coulomb interactions constrained by Wolfgang Pauli's exclusion principle, which forbade the occupancy of a quantum state by two electrons with the same spin.

The intrinsic angular momentum of an electron with two eigenvalues had been proposed by Pauli in 1924; Samuel Goudsmit and George Uhlenbeck demonstrated a year later that the spin angular momentum had a value of $1 / 2 \hbar$. The Pauli spin matrices representing the three components of spin angular momentum are

$$
\boldsymbol{s}=\left(\left[\begin{array}{ll}
0 & 1 \\
1 & 0
\end{array}\right],\left[\begin{array}{cc}
0 & -\mathrm{i} \\
\mathrm{i} & 0
\end{array}\right],\left[\begin{array}{cc}
1 & 0 \\
0 & -1
\end{array}\right]\right) \hbar / 2
$$

The corresponding electronic magnetic moment was the Bohr magneton,

$$
\mu_{\mathrm{B}}=\mathrm{e} \hbar / 2 \mathrm{~m}_{\mathrm{e}}
$$

or $9.274 \times 10^{-24} \mathrm{Am}^{2}$, twice as large as the moment associated with a unit of orbital angular momentum in Bohr's model of the atom. The gyromagnetic ratio of magnetic moment to angular momentum for the electron spin is $\gamma \approx \mathrm{e} / \mathrm{m}_{\mathrm{e}}$, so the Larmor precession frequency $\mathrm{e} B / 2 \pi \mathrm{m}_{\mathrm{e}}$ for the electron is $28 \mathrm{GHzT}^{-1}$. 
The problem of the electron's magnetism was finally resolved by Paul Dirac in 1928 when he succeeded in writing Schrödinger's equation in relativistically invariant form, obtaining the non-relativistic electron spin in terms of the $2 \times 2$ Pauli matrices. Together with Dirac, Werner Heisenberg formulated the exchange interaction represented by the famous Heisenberg Hamiltonian

$$
\mathcal{H}=-2 \mathcal{J} \boldsymbol{S}_{i} . \boldsymbol{S}_{j}
$$

to describe the coupling between the vector spins $S_{i}$ and $S_{j}$ of two nearby manyelectron atoms $i$ and $j$. The spin vectors $S$ are the spin angular momenta in units of $\hbar$. The value of the exchange integral $\mathcal{J}$ was closely related to Weiss's molecular field coefficient $n_{\mathrm{W}}$ and depends strongly on interatomic distance. It can be positive, if it tends to align the two spins parallel (ferromagnetic exchange), or negative if it tends to align the pair antiparallel (antiferromagnetic exchange). The value of $S$ is obtained from the first of the three rules, discussed below, that were formulated by Friedrich Hund around 1927 for finding the ground state of a multi-electron atom. The exchange interactions among the electrons of the same atom are much stronger than those between the electrons of adjacent atoms given by Eq. (15). The fundamental insight that magnetic coupling of electronic spins is governed by electrostatic coulomb interactions, subject to the symmetry constraints of quantum mechanics, was the key needed to unlock the mysteries of ferromagnetism. Exchange is discussed in > Chap. 2, "Magnetic Exchange Interactions."

The magnetic moment of an atom or ion is the sum of two contributions. One arises from the intrinsic spin angular momentum of the atomic electrons. The other comes from their quantized orbital angular momentum. The moments associated with each type of angular momentum have to be summed according to the rules of quantum mechanics. The moment associated with $1 / 2 \hbar$ of spin angular momentum is practically identical to that associated with $\hbar$ of orbital angular momentum, namely, one Bohr magneton in each case. The quantum theory of magnetism is therefore the quantum theory of angular momentum. Hund's rules were an empirical prescription for determining the total angular momentum of the many-electron ground state of electrons belonging to the same atom or ion. Firstly, the rule is to maximize the spin angular momentum $S$ while respecting the Pauli principle that no two electrons can be in the same quantum state. Secondly, the orbital angular momentum $L$ is maximized, consistent with the value of $S$, and thirdly the spin and orbital momenta are coupled together to form the total angular momentum $J=L \pm S$, according to whether the electronic shell is more or less than half full. The total magnetic moment (in units of $\mu_{\mathrm{B}}$ ) is then related to the total angular momentum (in units of $\hbar$ ) by a numerical Landé $\mathrm{g}$-factor, which is 1 for a purely orbital moment and 2 for pure spin.

The spin-orbit coupling, which arises in the atom from motion of the electron in the electrostatic potential of the charged nucleus and gives rise to Hund's third rule, is another key interaction. Of fundamentally relativistic character, it emerges naturally from Dirac's relativistic quantum theory of the electron, and it turns out to be at the root of many of the most interesting phenomena in magnetism, including 
magneto-optics, magnetocrystalline anisotropy, and the spin Hall effect. The spinorbit interaction for a magnetic ion is represented by the Hamiltonian $\Lambda \boldsymbol{L} . \boldsymbol{S}$, where $\boldsymbol{L}$ is the orbital angular momentum of the many-electron atom in units of $\hbar$ and $\Lambda$ is the atomic spin-orbit coupling constant. Like the exchange constant $\mathcal{J}, \Lambda$ has dimensions of energy.

Felix Bloch in 1930 described the spin waves that are the quantized elementary excitations of a ferromagnetic array of atoms whose spins are coupled by Heisenberg exchange. These excitations have an angular frequency $\omega$ and a wavevector $\boldsymbol{k}$ that are related by the dispersion relation $\hbar \omega=D k^{2}$, where $D$ is the spin wave stiffness constant. It is proportional to $\mathcal{J}$.

The first quantum theories of magnetism regarded the electrons as localized on the atoms or ions, but an alternative magnetic band theory of ferromagnetic metals was developed by John Slater and Edmund Stoner in the 1930s. It accounted for the non-integral, delocalized spin moments found in $\mathrm{Fe}, \mathrm{Co}$, and $\mathrm{Ni}$ and their alloys, although the theory in its original form greatly overestimated the Curie temperatures. The delocalized, band electron model of Slater and the localized, atomic electron model of Heisenberg were two distinct paradigms for the theory of magnetism that persisted until sophisticated computational methods for treating the many-body interelectronic correlations in the ground state of multi-electron atoms were devised toward the end of the twentieth century. The differences between the two approaches are epitomized in the calculation of the paramagnetic susceptibility. Pauli found a small temperature-independent susceptibility resulting from Fermi-Dirac statistics for delocalized electrons, whereas Léon Brillouin had used Boltzmann statistics and the Bohr model to derive the Curie law susceptibility of an array of atoms with localized electrons.

The sixth Solvay Conference, held in Brussels in October 1930 (Fig. 9), was devoted to magnetism [21]. It followed four years of brilliant discoveries in theoretical physics, which set out the modern electronic theory of condensed matter. Yet the immediate impact on the practical development of functional magnetic materials was surprisingly slight. Dirac there made the perceptive remark "The underlying physical laws necessary for the mathematical theory of a large part of physics and the whole of chemistry are completely known, and the difficulty is only that the exact application of these laws leads to equations much too complicated to be soluble."

\section{Magnetic Phenomenology}

In view of the immense computational challenge posed by many-body electron physics in 1930, a less fundamental theoretical approach was needed. Louis Néel pursued a phenomenological approach to magnetism with notable success, oblivious to the triumphs of quantum mechanics. His extension of the Weiss theory to two equal but oppositely aligned magnetic sublattices led him to the idea of antiferromagnetism in his 1932 doctoral thesis. This hidden magnetic order 


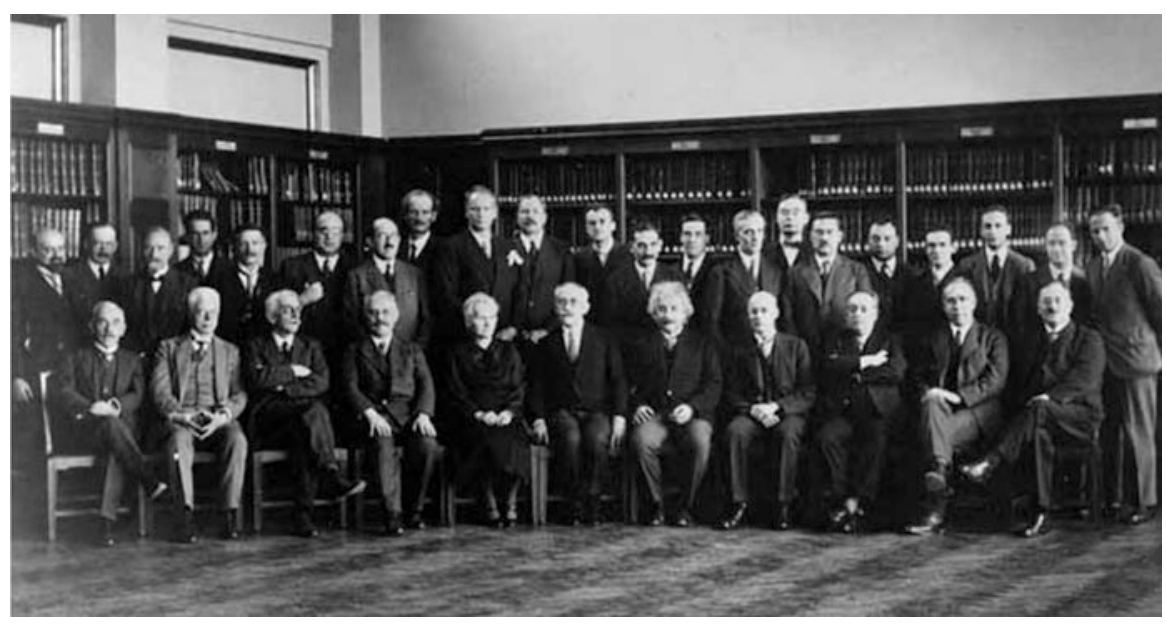

Fig. 9 The 1930 Solvay Conference on Magnetism Back row: Herzen, Henriot, Verschaffelt, Manneback, Cotton, Errera, Stern, Piccard, Gerlach, Darwin, Dirac, Bauer, Kapitza, Brioullin, Kramers, Debye, Pauli, Dorfman, van Vleck, Fermi, Heisenberg. Front row: de Donder, Zeeman, Weiss, Sommerfeld, Curie, Langevin, Einstein, Richardson, Cabrera, Bohr, de Haas

awaited the development of neutron scattering in the 1950 s before it could be directly revealed, initially for $\mathrm{MnO}$. Néel went on to explain the ferrimagnetism of oxides such as magnetite, $\mathrm{Fe}_{3} \mathrm{O}_{4}$, the main constituent of lodestone, in terms of two unequal, antiferromagnetically coupled sublattices. The three most common types of magnetic order, and their temperature dependences, are illustrated in Fig. 10.

The spinel $\left(\mathrm{MgAl}_{2} \mathrm{O}_{4}\right)$ structure of magnetite has an A sublattice of $8 a$ sites with fourfold tetrahedral oxygen coordination and twice as many $16 d$ sites with sixfold octahedral coordination forming a B sublattice. The spinel structure is illustrated in Fig. 14 where the $8 a$ sites are at the centers of the blue tetrahedra, which have oxygen ions at the four corners, and the $16 d$ sites are at the centers of the brown octahedra, which have six oxygen ions at the corners. The numbers of each type of site in the unit cell are indicated by the labels. The $16 d$ sites in magnetite are occupied by a mixture of ferrous $\mathrm{Fe}^{2+}$ and ferric $\mathrm{Fe}^{3+}$ ions with electronic configurations $3 d^{5}$ and $3 d^{6}$ and spin moments of $5 \mu_{\mathrm{B}}$ and $4 \mu_{\mathrm{B}}$, respectively, whereas the $8 a$ sites are occupied by oppositely aligned $\mathrm{Fe}^{3+}$ ions. This yields a net spin moment of $4 \mu_{B}$ per formula $\left(0.48 \mathrm{MAm}^{-1}\right)$ - a quantitative explanation of the magnetism of the archetypical magnet in terms of lattice geometry and the simple rule that each unpaired electron contributes a spin moment of one Bohr magneton. Néel added two new categories of magnetic substances - antiferromagnets and ferrimagnets - to Faraday's original three. Their magnetic ordering temperatures are known as antiferromagnetic or ferrimagnetic Néel temperatures. The ferrimagnetic one is also called a Curie point. 

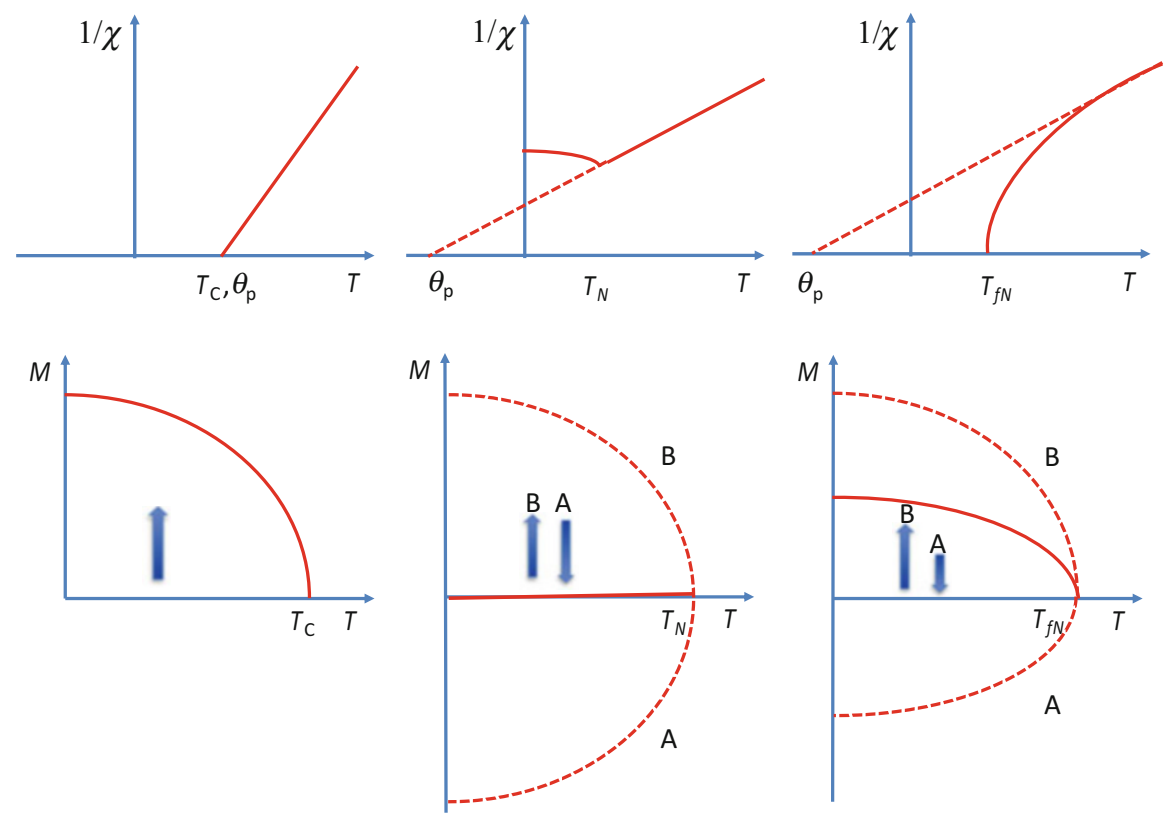

Fig. 10 Schematic temperature dependences of the inverse susceptibility (top) and (sub)lattice magnetization (bottom) of a ferromagnet (left), an antiferromagnet (center), and a ferrimagnet (tight)

\section{Micromagnetism}

For many practical purposes, it is possible to follow in the footsteps of Néel, sidestepping the complications engendered by the atomic and electronic basis of magnetism, and regard magnetization as a continuous vector in a solid continuum [13], as people have for about 200 years. The iconic hysteresis loop $\boldsymbol{M}(\boldsymbol{H})$ (Fig. 8) is the outcome of a metastable structure of domains of uniformly magnetized ferromagnetic Weiss domains separated by narrow domain walls between domains magnetized in different directions. The structure depends on the thermal and magnetic history of a particular sample. Aural evidence for discontinuous jumps in the size of the domains as the magnetization was saturated was first heard by Heinrich Barkhausen in 1919 with the help of a pickup coil wound around some ferromagnetic wires, a rudimentary amplifier, and a loudspeaker. Then in 1931 the domains were directly visualized by Francis Bitter using a microscope focused on a polished sample surface and a colloidal suspension of magnetite particles that were drawn by the stray field to the domain walls. These colloids, known as ferrofluids, behave like ferromagnetic liquids.

The idea of a domain wall as a region where the magnetization rotates progressively from one direction to the opposite one in planes parallel to the wall was introduced by Felix Bloch in 1932. His walls create no bulk demagnetizing 
(a)

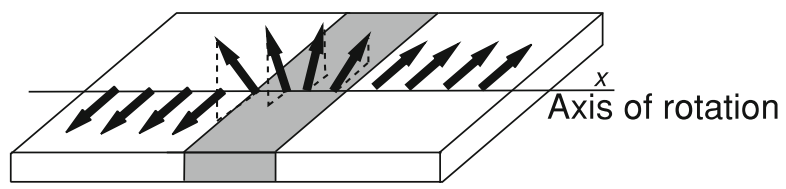

(b)

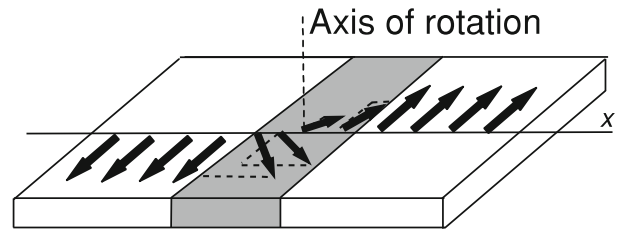

Fig. 11 Two types of $180^{\circ}$ domain walls: a) the Bloch wall and b) the Néel wall

field and cost little magnetostatic energy because $\nabla . \boldsymbol{M}=0$; the magnetization in each plane is uniform, and there is no component perpendicular to the planes (see Eq. 9). The exchange energy cost, written in the continuum approximation as $A(\nabla \boldsymbol{M})^{2}$ where $A \propto \mathcal{J}$, is balanced by the anisotropy energy cost associated with the magnetization in the wall that is misaligned with respect to a magnetic easy axis of the crystal. Magnetic anisotropy is introduced below, and it is discussed in detail in - Chap. 3, "Anisotropy and Crystal Field." A Néel domain wall, where the magnetization rotates in a plane perpendicular to the wall so that $\nabla . \boldsymbol{M} \neq 0$ in the bulk, but there is no surface magnetic charge, is higher in energy except in thin films. The two types of wall are illustrated in Fig. 11.

In principle, the sum of free energy terms associated with exchange, anisotropy, and magnetostatic interactions, together with the Zeeman energy in an external field, could be minimized to yield the $\boldsymbol{M}(\boldsymbol{H})$ loop and the overall domain structure of any solid. Further terms can be added to take into account the effects of imposed strain and spontaneous magnetostriction. In practice, however, crystal defects such as grain boundaries spoil the continuum picture and can exert a crucial influence on the walls. It is then necessary to resort to models to develop an understanding of hysteresis.

The basic theory of micromagnetism was developed by William Fuller Brown in 1940 [13]. The magnetostatic interaction between the magnetic dipoles that constitute the magnetization is a dominant factor. The dipole fields fall off as $1 / r^{3}$ (Eq. 2), providing a long-range interaction unlike exchange, which is short-range because it depends on an overlap between electronic wavefunctions that decays exponentially with interatomic spacing. This is why weak magnetostatic interactions that are of order $1 \mathrm{~K}$ for a pair of ions are able to compete on a mesoscopic length scale with the much stronger exchange interactions of electrostatic origin that can be of order $100 \mathrm{~K}$ to control the domain structure of a given ferromagnetic sample.

Magnetocrystalline anisotropy is represented phenomenologically in the theory by terms in the energy that depend on the orientation of $\boldsymbol{M}$ with respect to the local crystal axes. The electrostatic interaction of localized atomic electrons with the potential created by all the other atoms in the crystal is known as the crystal field 
interaction; the effect of chemical bonding with the ligands of an atom is the ligand field interaction. The two effects are comparable in magnitude for $3 d$ ions [22]. Magnetocrystalline anisotropy arises from the interplay of the crystal/ligand field and spin-orbit coupling. The simplest case is for uniaxial (tetragonal, hexagonal, rhombohedral) crystals, where the leading term in the energy density is of the form

$$
E_{\mathrm{a}}=K_{1} \sin ^{2} \theta+\ldots
$$

where $\theta$ is the angle between $\boldsymbol{M}$ and the symmetry axis. Two opposite easy directions lie along the crystal axis if the anisotropy constant $K_{1}$ is positive, but there are many easy directions lying in an easy plane perpendicular to the crystal axis $(\theta=\pi / 2)$ when $K_{1}$ is negative. Anisotropy arises also from overall sample shape, due to the demagnetizing energy $1 / 2 M H_{\mathrm{d}}$, which gives another contribution in $\sin ^{2} \theta$ that depends on the demagnetizing factor $\mathcal{N}$ with

$$
K_{1}^{\mathrm{sh}}=\frac{1}{4} \mu_{0} M_{\mathrm{s}}^{2}(1-3 \mathcal{N})
$$

where $M_{\mathrm{S}}$ is the spontaneous magnetization. There is obviously no shape anisotropy for a sphere, which has $\mathcal{N}=1 / 3$. An expression equivalent to (16) at the atomic scale is $\varepsilon_{\mathrm{a}}=D_{\mathrm{a}} \sin ^{2} \theta$, where $D_{\mathrm{a}} / \mathrm{k}_{\mathrm{B}} \sim 1 \mathrm{~K}$. The magnitude of the crystal field energy is comparable to the magnetostatic energy, but it is much smaller than the exchange energy in practical magnetic materials. It remains challenging to calculate $K_{1}$ or $D_{\mathrm{a}}$ precisely in metals.

An instructive paradox arising from Brown's micromagnetic theory is his result that the coercivity $H_{\mathrm{c}}$ of a perfect, defect-free ferromagnetic crystal lattice must exceed the anisotropy field $H_{\mathrm{a}}=2 K_{1} / \mu_{0} M_{\mathrm{s}}$. In practice $H_{\mathrm{c}}$ is rarely as much as a fifth of $H_{\mathrm{a}}$. The explanation is that no real lattice is ever free of defects, which act as sites for the nucleation of reverse domains or as pinning centers for domain walls. The sequence of metastable states represented on the hysteresis loop is generally dominated by asperities and lattice defects that are very challenging to characterize in any real macroscopic sample. Control of these defects in modern permanent magnets having $H_{\mathrm{c}} \gtrsim M_{\mathrm{s}}$ has been as much a triumph of metallurgical art as physical theory. Micromagnetism is the subject of $\triangleright$ Chap. 7, "Micromagnetism."

\section{Magnetic Materials}

The traditional magnetic materials were alloys of the ferromagnetic metals, Fe, $\mathrm{Co}$, and Ni. The metallurgy and magnetic properties of these alloy systems were the focus of investigations of technical magnetism in the first half of the twentieth century, when useful compositions were developed such as Permendur, $\mathrm{Fe}_{50} \mathrm{Co}_{50}$, the alloy with the highest magnetization (1.95 $\mathrm{MAm}^{-1}$ ); Permalloy $\mathrm{Fe}_{20} \mathrm{Ni}_{80}$, which has near-zero anisotropy and magnetostriction, together with very high relative permeability $\left(\mu_{\mathrm{r}}=(1+\chi) \approx 10^{5}\right)$; and Invar $\mathrm{Fe}_{64} \mathrm{Ni}_{36}$ a composition with near- 

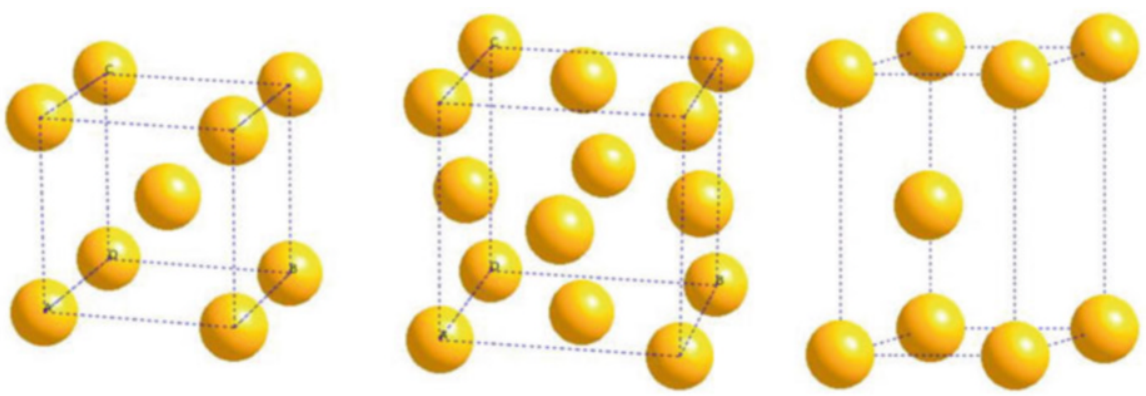

Fig. 12 Unit cells of the ferromagnetic elements $\mathrm{Fe}$ (body-centered cubic, left), $\mathrm{Ni}$ (face-centered cubic, center), and Co, Gd (hexagonal close-packed, right) [29], with kind permission from Cambridge University Press

zero thermal expansion around room temperature. The early investigations are well summarized in Bozorth's 1950 monograph [23]. The fourth ferromagnetic element at room temperature is the rare earth gadolinium. The crystal structures of these elemental ferromagnets are illustrated in Fig. 12.

An important practical advance in the story of permanent magnet development was the thermal processing of a series of Al-Ni-Co-Fe alloys, the Alnico magnets, that was initiated in Japan in 1932 by Tokushichi Mishima. Their coercivity relied on achieving a nanostructure of aligned acicular (needle-like) regions of Co-Fe in a matrix of nonmagnetic Ni-Al. It was the shape of the ferromagnetic regions that gave the alloys some built-in magnetic anisotropy (Eq. 17), but it still had to be supplemented with global shape anisotropy by fabricating the Alnico into a bar or horseshoe in order to avoid self-demagnetization. The mastery of coercivity that was acquired over the course of the twentieth century (Fig. 13) was spectacular, and burgeoning applications in technical magnetism of soft and hard magnetic materials were the direct consequence. The terms "soft" and "hard" were derived originally from the magnetic steels that were used in the nineteenth century. The most useful figure of merit for the hard, permanent magnets is the maximum energy product $|B H|_{\max }$, equal to twice the energy in the stray field produced by a unit volume of magnet. The SI unit is $\mathrm{kJm}^{-3}$. Energy product doubled every 12 years for most of the twentieth century, thanks to the discovery in the 1960s of rare earth cobalt intermetallic compounds and the discovery of new rare earth ironbased materials in the 1980s. Comparable progress with decreasing hysteresis losses in soft, electrical steels continued to the point where they became a negligible fraction of the resistive losses in the copper windings of electromagnetic energy converters. Ultrasoft amorphous magnetic glasses were developed in the 1970s. Applications of soft and hard magnetic materials are discussed in $>$ Chaps. 29, "Soft Magnetic Materials and Applications," and > 28, "Permanent Magnet Materials and Applications" respectively.

A good working knowledge of the quantum mechanics of multi-electron atoms and ions had been developed by the middle of the twentieth century, mainly from 


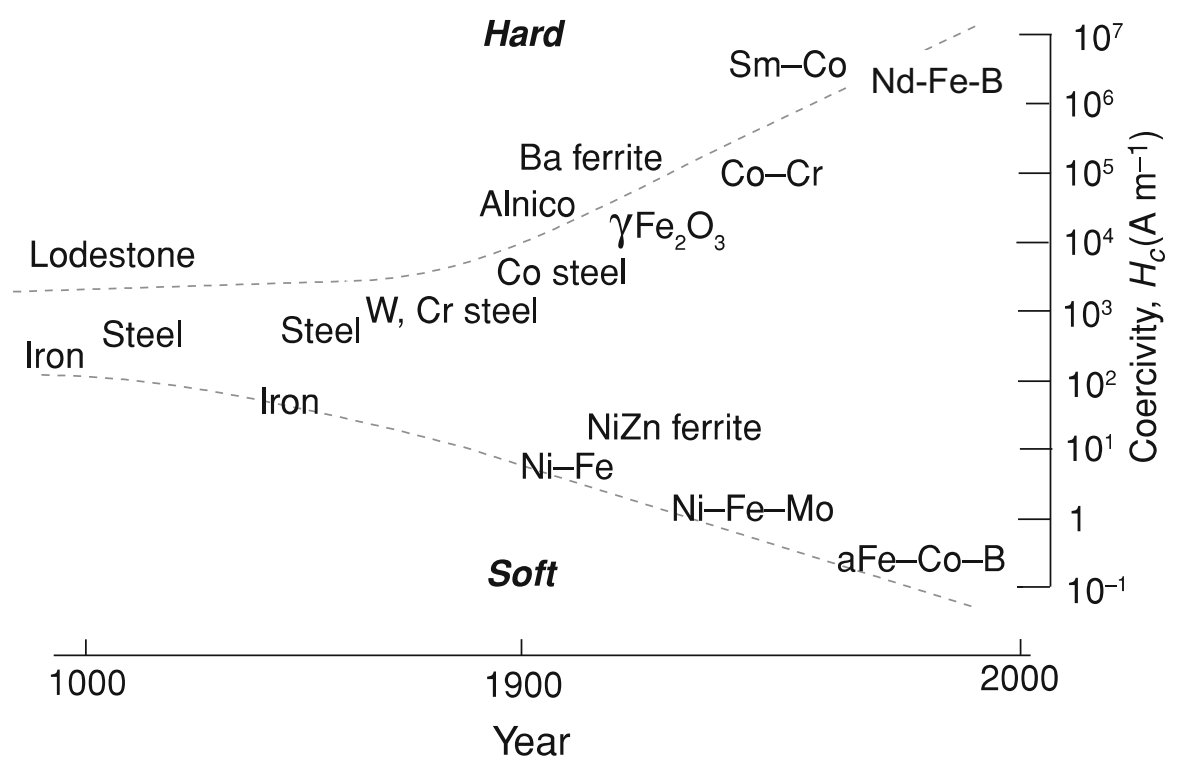

Fig. 13 The development of coercivity over the ages and in the twentieth century

observations of optical spectra and the empirical rules formulated by Hund to specify the ground state $L, S$, and $J$ multiplet, which is the one of interest for magnetism. All this led naturally to a focus on the localized electron magnetism found in the $3 d$ and $4 f$ series of the periodic table. For $3 d$ ions in solids, the ionic moment is essentially that arising from the unpaired electron spins left after filling the orbitals according to the Pauli principle and Hund's first rule. The orbital moment expected from the second rule is quenched by the crystal field, which impedes the orbital motion so that it barely contributes to the ionic magnetism. But the crystal field is weaker for the $4 f$ elements in solids, whether insulating or metallic, and the magnetism is more atomic-like with spin and orbital contributions coupled by the spin-orbit interaction according to Hund's third rule to yield the total angular momentum $\boldsymbol{J}$.

Microscopic quantum theory began to play a more important part in magnetic materials development after the 1970s with the advent of rare earth permanent magnets $\mathrm{SmCo}_{5}$ and especially $\mathrm{Nd}_{2} \mathrm{Fe}_{14} \mathrm{~B}$, when an understanding of the intrinsic, magnetocrystalline anisotropy in terms of crystal field theory and spin-orbit coupling began at last to make a contribution to the design of new permanent magnet materials.

\section{Magnetic Oxides}

The focus on localized electron magnetism in the 1950s and 1960s led to systematic investigations of exchange interactions in insulating compounds where the spin 
moments of magnetic $3 d$ ions are coupled by indirect overlap of their wavefunctions via an intervening nonmagnetic anion, usually $\mathrm{O}^{2-}$. A systematic empirical understanding of the dependence of these superexchange interactions on electron occupancy and bond angle emerged in the work of Junjiro Kanamori and John Goodenough [24], based on the many new magnetic compounds that were being fabricated at that time. There is a multitude of solid solutions between end-members, with extensive opportunities to tune magnetic properties by varying the chemical compositions of oxide families such as ferrites [25]. Superexchange, like direct exchange in the ferromagnetic $3 d$ elements, depends on the overlap of wavefunctions of adjacent atoms and decays exponentially with interatomic distance.

The magnetite family of cubic spinel ferrites $\mathrm{M}^{2+} \mathrm{Fe}_{2}^{3+} \mathrm{O}_{4}$ was the first to be thoroughly investigated, with $\mathrm{M}=\mathrm{Mg}, \mathrm{Zn}, \mathrm{Mn}, \mathrm{Fe}, 2 / 3 \mathrm{Fe}^{3+}\left(\gamma \mathrm{Fe}_{2} \mathrm{O}_{3}\right), \mathrm{Co}$, or $\mathrm{Ni}$. Ferrimagnetic Neél temperatures of these ferrites range from 700 to $950 \mathrm{~K}$, although spinel itself $\left(\mathrm{MgAl}_{2} \mathrm{O}_{4}\right)$ is nonmagnetic. Several of the insulating compounds with $\mathrm{Mn}, \mathrm{Ni}$, and $\mathrm{Zn}$ are suitable as soft magnetic materials for audio- or radiofrequency applications. Other important families investigated at that time were garnets, perovskites, and hexagonal ferrites. The garnet ferrites $\mathrm{R}_{3}^{3+} \mathrm{Fe}_{5}^{3+} \mathrm{O}_{12}$ have a large cubic unit cell containing 160 ions, with ferrimagnetically aligned ferric iron in both tetrahedral $24 d$ and octahedral $16 a$ sites, and large $\mathrm{R}^{3+}$ ions in eightfold oxygen coordination in deformed cubal $24 c$ sites. $\mathrm{R}$ may be any rare earth element, including $\mathrm{Y}$, which forms yttrium iron garnet (YIG), $\mathrm{Y}_{3} \mathrm{Fe}_{5} \mathrm{O}_{12}$, a superlative microwave material that exhibits ultra-low magnetic losses on account of its insulating character. The net magnetic moment of YIG is $5 \mu_{\mathrm{B}}$ per formula unit. Substituting magnetic rare earths in the structure provides an opportunity to study superexchange between $3 \mathrm{~d}$ and $4 f$ ions. That interaction is weak, and the $4 f$ ions couple antiparallel to the $24 d$ site iron, but their sublattice magnetization decays much faster with temperature, giving rise to the possibility of a compensation temperature, where the net magnetization of the two ferrimagnetic sublattices crosses zero at a temperature below the ferrimagnetic Neél point. The compensation temperature of $\mathrm{Gd}_{3} \mathrm{Fe}_{5} \mathrm{O}_{12}$, for example, is $290 \mathrm{~K}$, whereas its ferrimagnetic Néel point is at $560 \mathrm{~K}$, a typical value for the whole rare earth iron garnet series.

Another important oxide family, the hexagonal ferrites especially $\mathrm{M}_{2} \mathrm{Fe}_{12} \mathrm{O}_{19}$, where $\mathrm{M}=\mathrm{Ba}^{2+}$ or $\mathrm{Sr}^{2+}$, have uniaxial anisotropy and crystallize in the magnetoplumbite structure. There are four $\mathrm{Fe}^{3+}$ sites in the structure, including a fivefold $2 b$ site with trigonal symmetry where the threefold axis is parallel to the c-axis of the hexagonal unit cell. The net ferrimagnetic moment is $20 \mu_{\mathrm{B}}$ per formula unit, since eight iron ions belong to one sublattice and four to the other. The large nonmagnetic M cations occupy sites that would otherwise belong to a hexagonal close-packed oxygen lattice. The $2 b$ site contributes rather strong uniaxial anisotropy, and the anisotropy field of $1.4 \mathrm{MAm}^{-1}$ is more than three times the magnetization $(0.38$ $\mathrm{MAm}^{-1}$ ), making it possible in the early 1950 s to achieve coercivity comparable to the magnetization and manufacture cheap ceramic magnets in any desired shape, thereby overcoming the shape barrier that had impeded the development permanent magnets for a millennium. A million tonnes of these ferrite magnets is sold every year. 
The drawback of any oxide magnetic material is that its magnetization is never more than a third of that of metallic iron. This is unavoidable because most of the unit cell volume is occupied by large, nonmagnetic $\mathrm{O}^{2-}$ anions, with the high-spin ferric iron $\mathrm{Fe}^{3+}$ or other magnetic ions confined to the interstices in the oxygen lattice. To make matters worse, a ferrimagnetic structure reduces the magnetization further. There are relatively few ferromagnetic oxides; $\mathrm{CrO}_{2}$ is one example. It is not an insulator, but a half metal, with a gap in the minority-spin conduction band.

A search for insulating ferromagnetic oxides in the 1950s led to the investigation of $\mathrm{ABO}_{3}$ compounds with the perovskite structure. Here the magnetic $\mathrm{B}$ cations occupy the $1 a$ octahedral sites, and the nonmagnetic A cations occupy the 12-coordinated $1 b$ sites in the ideal cubic structure. It proved to be possible to obtain ferromagnetism provided the A cations are present in two different valence states. This works best in mixed-valence manganites [26], with composition $\left(\mathrm{La}_{0.7}^{3+} \mathrm{M}_{0.3}^{2+}\right) \mathrm{MnO}_{3}$ where $\mathrm{M}=\mathrm{Ba}, \mathrm{Ca}$, or Sr. The resulting mixture of $\mathrm{Mn}^{3+}\left(3 d^{4}\right)$ and $\mathrm{Mn}^{4+}\left(3 d^{3}\right)$ on $\mathrm{B}$ sites leads to electron hopping with spin memory from one $3 d^{3}$ core to another. This is the ferromagnetic double exchange interaction, envisaged by Clarence Zener in 1951. Similar electron hopping occurs for $\mathrm{Fe}^{2+}$ and $\mathrm{Fe}^{3+}$ in the octahedral sites of magnetite. A consequence is that the oxides, though ferromagnetic, are no longer insulating, and the Curie temperatures are not particularly high - they do not exceed $400 \mathrm{~K}$. A notable feature of the mixed-valence manganites, related to their hopping conduction, is the "colossal magnetoresistance" observed near the Curie point, where there is a broad maximum in the resistance that can be suppressed by applying a magnetic field of several tesla. All four oxide structures are presented in Fig. 14. They illustrate the importance of crystal chemistry for determining magnetic properties.
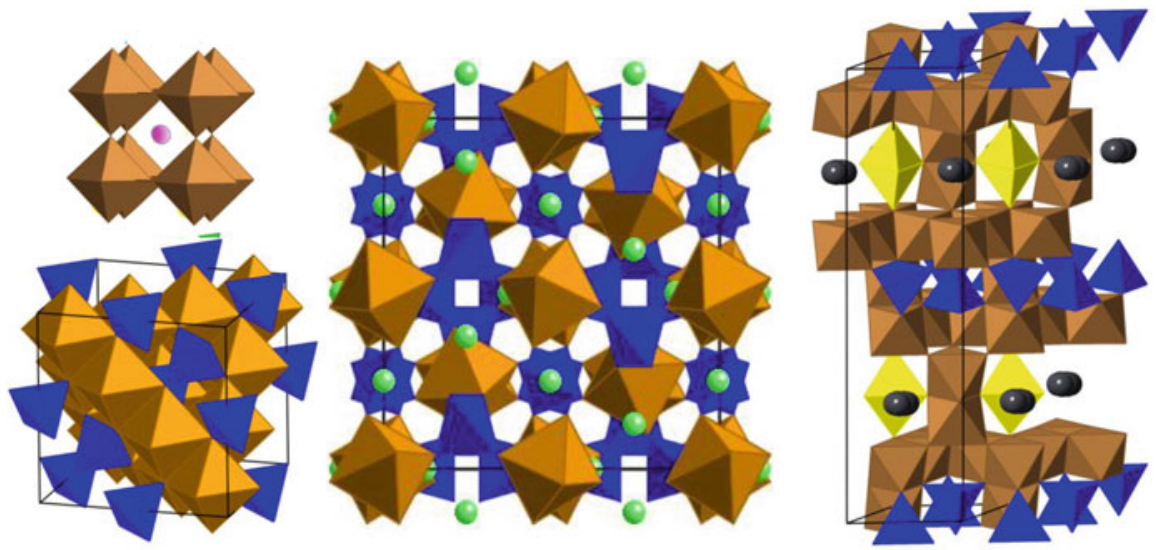

Fig. 14 Crystal structures of magnetic oxides: perovskite (top left), spinel (bottom left), garnet (center), magnetoplumbite (right). The oxygen coordination polyhedral around the magnetic cations (tetrahedrons, blue, or octahedrons, brown) is illustrated. The spheres are large nonmagnetic cations. Unit cells are outlined in black. Magnetoplumbite is hexagonal, and the others are cubic [31], with kind permission from APS 
Research on localized electron magnetism in oxides and related compounds has passed through three phases. Beginning with studies of polycrystalline ceramics from about 1950, single crystals were grown for specific physical investigations after about 1970, and then in the late 1980s, following the high-temperature superconductivity boom, came the growth and characterization of ferromagnetic and ferrimagnetic oxide thin films and first steps toward all-oxide spin electronics. A similar pattern was followed by sulfides, fluorides, and other magnetic compounds. All are discussed further in > Chap. 17, "Magnetic Oxides and Other Compounds."

\section{Intermetallic Compounds}

A rich class of functional magnetic materials is the intermetallic compounds of rare earth elements and transition metals. The atomic volume ratio of a $4 f$ to a $3 d$ atom is about three, so the alloys tend to be stoichiometric line compounds rather than solid solutions. The first of these was $\mathrm{SmCo}_{5}$, developed for permanent magnet applications in the USA in the mid-1960s by Karl Strnat. It was followed by $\mathrm{Sm}_{2} \mathrm{Co}_{17}$ in the early 1970 s, and then in 1983 came the announcement of the independent discovery of the first iron-based rare earth magnet, the ternary $\mathrm{Nd}_{2} \mathrm{Fe}_{14} \mathrm{~B}$, by Masato Sagawa in Japan and John Croat in the USA. This was a breakthrough because iron is cheaper and more strongly magnetic than cobalt. $\mathrm{Nd}_{2} \mathrm{Fe}_{14} \mathrm{~B}$ has since come to dominate the global high-performance magnet market, with an annual production in excess of 100,000 tonnes. The coercivity needed in these optimized rare earth permanent magnets is comparable to their magnetization, and the optimization of the microstructure of a new hard magnetic material to attain the highest possible energy product, which scales as $M_{\mathrm{s}}{ }^{2}$ but can never exceed $1 / 4 \mu_{0} M_{\mathrm{S}}{ }^{2}$, is a long empirical process. It generally takes many years to achieve a coercivity as high as $20-30 \%$ of the anisotropy field [28]. The battle to create the metastable hysteretic state that permits a permanent magnet to energize the surrounding space with a large stray field is never easy to win, and each material requires a different strategy.

The fundamental significance of these intermetallics and related interstitial compounds such as $\mathrm{Sm}_{2} \mathrm{Fe}_{17} \mathrm{~N}_{3}$ that were discovered in the 1990s is that crystal field theory and quantum mechanics were involved in their design. All have a uniaxial crystal structure with a single easy axis and strong magnetocrystalline anisotropy. Such anisotropy is a prerequisite for the substantial coercivity, $H_{\mathrm{c}} \gtrsim M_{\mathrm{s}}$ needed to overcome the shape barrier and create a magnet with any desired form.

The practical significance of the rare earth permanent magnets has been the appearance of a wide range of compact, energy-efficient electromagnetic energy converters that are being used in consumer products, electric vehicles, aeronautics, robotics, and wind generators.

Besides magnetocrystalline anisotropy, another potentially useful consequence of the spin-orbit interaction in rare earth intermetallics is their strong magnetostriction. The rare earth elements order magnetically at or below room temperature so, just as for the permanent magnets, it was necessary to form an intermetallic 

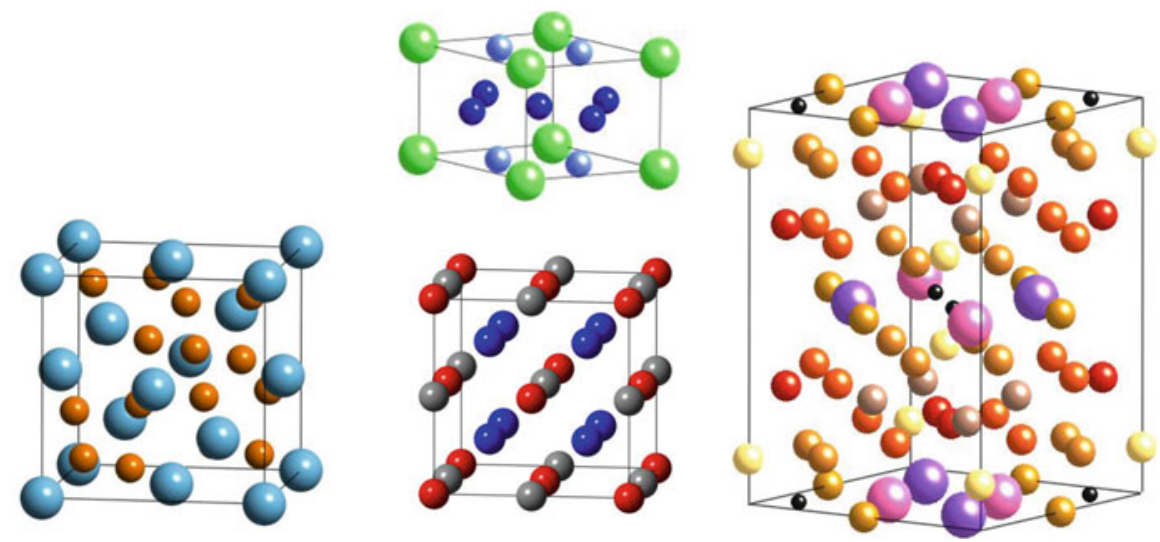

Fig. 15 Crystal structures of ferromagnetic intermetallic compounds: $\mathrm{YFe}_{2}$ (cubic, left) $\mathrm{SmCo}_{5}$ (hexagonal, top centre), $\mathrm{Co}_{2} \mathrm{MnSi}$ (cubic, bottom centre), $\mathrm{Nd}_{2} \mathrm{Fe}_{14} \mathrm{~B}$ (tetragonal, right). Fe and $\mathrm{Co}$ $\mathrm{Mn}$ are the small brown/red, blue, and scarlet spheres. Rare earths are the large spheres. Si and B are grey and black

compound with iron or cobalt to obtain a functional material with a useful Curie temperature that should be substantially greater than room temperature to ensure adequate magnetic stability. A functional magnetostrictive material has to be magnetically soft, and this was achieved in the $\mathrm{RFe}_{2}$ rare earth Laves phase compounds by Arthur Clark in 1984, who combined Dy and Tb, which have the same sign of magnetostriction, but compensating anisotropy of opposite sign, in the cubic alloy $\left(\mathrm{Tb}_{0.3} \mathrm{Dy}_{0.7}\right) \mathrm{Fe}_{2}$, known as Terfenol-D. Single crystals exhibited Joulian magnetostriction of up to 2000 parts per million (ppm), a hundred times greater than Joule had measured 150 years earlier in pure iron [16] (see $>$ Chaps. 28, "Permanent Magnet Materials and Applications," and $>11$, "Magnetostriction and Magnetoelasticity").

Magnetically soft rare earth intermetallics are also of interest as magnetocaloric materials for solid-state refrigeration when their Curie point is close to room temperature (see $\triangleright$ Chap. 30, "Magnetocaloric Materials and Applications"). Some crystal structures of rare earth intermetallics are shown in Fig. 15.

Among the other intermetallic families, the ordered body-centered cubic Heusler families of $\mathrm{X}_{2} \mathrm{YZ}$ or $\mathrm{XYZ}$ alloys are notable in that they include a wide variety of magnetically ordered compounds, such as the magnetic shape-memory alloy $\mathrm{NiMnSb}$ or the half-metallic ferromagnet $\mathrm{Co}_{2} \mathrm{MnSi}$, which, like $\mathrm{CrO}_{2}$, has a gap at the Fermi level for minority-spin electrons. Information on a great many metallic magnetic materials is collected in $\triangleright$ Chap. 4, "Electronic Structure: Metals and Insulators." 


\section{Model Systems}

Magnetism has proved to be a fertile proving ground for condensed matter theory. The first mean-field theory was Weiss's molecular field of magnetism, later generalized by Lev Landau in the USSR in 1937. There followed more sophisticated theories of phase transitions, with magnetism providing much of the data to support them. The single-ion anisotropy of rare earth ions due to the local crystal field reduces the effective dimensionality of the magnetic order parameter from three to two for easy-plane ( $x y)$ anisotropy or from three to one for easy-z-axis (Ising) anisotropy. Magnetically ordered compounds can be synthesized with an effective spatial dimension of one (chains of magnetic atoms), two (planes of magnetic atoms), or three (networks of magnetic atoms), as well as ladders and isolated motifs. Magnetism has provided a treasury of materials that show continuous phase transitions as a function of temperature or quantum phase transitions at zero temperature as a function of pressure or magnetic field, as well as topological phases such as the two-dimensional $x y$ model, investigated by David Thouless, Michael Kosterlitz, and Duncan Haldane. It is frequently possible to realize magnetic materials that embody the essential electronic or structural features of the theoretical models.

An early theoretical milestone was Lars Onsager's 1944 solution of the twodimensional Ising model, where spins are regarded as one-dimensional scalars that can take only values of \pm 1 . The behavior of more complex and realistic systems such as the three-dimensional Heisenberg model near its Curie temperature was solved numerically using the renormalization group technique developed by Kenneth Wilson in the 1970s. The ability to tailor model magnetic systems, with an effective spatial dimension of 1 or 2 due to their structures of chains or planes of magnetic ions and an effective spin dimension of 1,2, or 3 determined by magnetocrystalline anisotropy due to the combination of the crystal/ligand field and the spin-orbit interaction, was instrumental in laying the foundation of the modern theory of phase transitions. The theory is based on universality classes where power-law temperature variations of the order parameter and its thermodynamic derivatives with respect to temperature or magnetic field in the vicinity of the phase transition are characterized by numerical critical exponents that depend only by the dimensionality of the space and the magnetic order parameter.

Another fecund line of enquiry was "Does a single impurity in a metal bear a magnetic moment?" This was related to Jun Kondo's formulation of a problem concerning the scattering of electrons by magnetic impurities in metals and its eventual solution in 1980. In the presence of antiferromagnetic coupling between an impurity and the conduction electrons of a metallic host, the combination enters a nonmagnetic ground state below the Kondo temperature $T_{\mathrm{K}}$. The Kondo effect is characterized by a minimum in the electrical resistivity. The study of magnetic impurities in metals focused attention on the relation between magnetism and electronic transport, which has proved extremely fruitful, leading to several Nobel Prizes and the emergence in the 1990s of spin electronics. 
The exchange interaction between two dilute magnetic impurities in a metal is long-range, decaying as $1 / r^{3}$ while oscillating in sign between ferrromagnetic and antiferromagnetic, where $r$ is their separation. The following is the RudermanKittel-Kasuya-Yosida (RKKY) exchange interaction

$$
\mathcal{J}(r)=\mathrm{a} \mathcal{J}_{\mathrm{sd}}^{2}(\sin \xi-\xi \cos \xi) / \xi^{4}
$$

where a is a constant, $\mathcal{J}_{\text {sd }}$ is the exchange coupling between the localized impurity and the conduction electrons, and $\xi$ is twice the product of $r$ and the Fermi wavevector. It was studied intensively in the 1970s in dilute alloys such as $\mathbf{A u F e}$ or $\mathbf{C u M n}$, known as spin glasses (the host is in bold type, and the impurity in italics). The impurity in these hosts retains its moment at low temperatures, and the RKKY exchange coupling $\mathcal{J}(\nabla)$ between a pair of spins is as likely to be ferromagnetic (positive) as antiferromagnetic (negative). The impurity spins freeze progressively in random orientations around a temperature $T_{\mathrm{f}}$ that is proportional to the magnetic concentration. The nature of this transition to the frozen spin glass state was exhaustively debated. A related issue, the long-range exchange interactions associated with the ripples of spin polarization created by a magnetic impurity in a metal, led to an understanding of complex magnetic order in the rare earth metals ( $>$ Chap. 14, "Magnetism of the Elements").

The magnetism of electronic model systems such as a chain of $1 s$ atoms with an on-site coulomb repulsion $U$ when two electrons occupy the same site, formulated by John Hubbard in 1963, has proved to be remarkably complex. Control parameters in the Hubbard model are the band filling and the ratio of $U$ to the bandwidth, and they lead to insulating and metallic, ferromagnetic, and antiferromagnetic solutions.

\section{Amorphous Magnets}

An important question, related to the dilute spin glass problem, was what effect does atomic disorder have on magnetic order and the magnetic phase transition in magnetically concentrated systems? Here a dichotomy emerges between ferromagnetic and antiferromagnetic interactions. The answer for materials with ferromagnetic exchange and a weak local electrostatic (crystal field) interaction is that the atomic disorder has little effect.

Techniques for rapidly cooling eutectic melts at rates of order $10^{6} \mathrm{Ks}^{-1}$ developed around 1970 produced a family of useful amorphous ferromagnetic alloys based on $\mathrm{Fe}, \mathrm{Co}$, and $\mathrm{Ni}$, with a minor amount of metalloid such as $\mathrm{B}, \mathrm{P}$, or $\mathrm{Si}$. These metallic glasses, frequently in the form of thin ribbons obtained by melt spinning, were magnetically soft and proved that ferromagnetic order could exist without a crystal lattice. There are no crystal axes, and weak local anisotropy due to the local electrostatic interactions averages out. The magnetic metallic glasses are mechanically strong and have found applications in transformer cores and security tags. 
Amorphous materials with antiferromagnetic interactions are qualitatively different. Whenever the superexchange neighbors in oxides or other insulating compounds form odd-membered rings, these interactions are frustrated. No collinear magnetic configuration is able to satisfy them all. In crystalline antiferromagnets like rocksalt-structure $\mathrm{NiO}$, the partial frustration leads to a reduced Néel temperature, but in fully frustrated pyrochlore-structure compounds, for example, the Néel point is completely suppressed. In the amorphous state, however, frustration has a spatially random aspect, and it leads to random spin freezing with a tendency to antiferromagnetic nearest-neighbor correlations, known as speromagnetism.

The situation for amorphous rare earth intermetallic alloys, which are best prepared by prepared by rapid sputtering, is different. There the local anisotropy at rare earth sites is strong, and does not average out, but it tends to pin the rare earth moments to randomly oriented easy axes in directions that are roughly parallel to that of the local magnetization of the $3 d$ ferromagnetic sublattice for the light rare earths and roughly antiparallel to it for the heavy rare earths. The sign of the $3 d-4 f$ coupling changes in the middle of the series, so that amorphous Gd-Fe alloys, for example, are ferrimagnetic. ( $\mathrm{Gd}$ is the case where there are no orbital moment and no magnetocrystalline anisotropy on account of its half-filled, $4 f^{7}$ shell.)

Rapid quenching can also be used to produce nanocrystalline material with isotropic crystallite orientations of nanocrystals embedded in an amorphous matrix. Certain soft magnetic materials have such a two-phase structure. Nanocrystalline $\mathrm{Nd}-\mathrm{Fe}-\mathrm{B}$ produced by rapid quenching shows useful coercivity due to domain wall pinning at the $\mathrm{Nd}_{2} \mathrm{Fe}_{14} \mathrm{~B}$ nanocrystallite boundaries, but the remanence is only about half the saturation magnetization on account of the randomly directed easy axes of the tetragonal crystallites. The magnitude of the anisotropy and the nanoscale dimension are critical for the averaging that determines the magnetic properties.

\section{Magnetic Fine Particles}

An early approach to the difficult problem of calculating hysteresis was to focus on magnetization reversal in single-domain particles that were too small to benefit from any reduction in their energy by forming a domain wall. Edmund Stoner and Peter Wohlfarth proposed an influential model in 1948. The particles were assumed each to have a single anisotropy axis, and the reverse field parallel to the axis necessary for magnetic reversal was the anisotropy field $H_{\mathrm{a}}=2 K_{\mathrm{u}} / \mu_{0} M_{\mathrm{s}}$, potentially a very large value. There was no coercivity when the field was applied perpendicular to the axis. Insights arose from the substantial deviation of real systems from the idealized Stoner-Wohlfarth model.

Meanwhile, the following year Néel, seeking to understand the remanent magnetism and hysteresis of baked clay and igneous rocks, proposed a model of thermally driven fluctuations of the magnetization of nanometer-sized ferromagnetic particles of volume $V$, a phenomenon known as superparamagnetism. The fluctuation time depended exponentially on the ratio of the energy barrier to magnetic 
reversal reversal $\Delta \approx K_{\mathrm{u}} V$ to the thermal energy $\mathrm{k}_{\mathrm{B}} T$. Here $K_{\mathrm{u}}$ is the uniaxial anisotropy (Eq. 16) of shape or magnetocrystalline origin. The expression for the time $\tau$ that elapses before a magnetic reversal is

$$
\tau=\tau_{0} \exp \left(\Delta / \mathrm{k}_{\mathrm{B}} T\right)
$$

where the attempt frequency $1 / \tau_{0}$ was taken to be the natural resonance frequency, $\sim 10^{9} \mathrm{~Hz}$. When the particles are superparamagnetic, the magnetization of particles smaller than a critical size fluctuates rapidly above a critical blocking temperature. The magnetization at lower temperatures, or for larger particles, does not fluctuate on the measurement timescale, and the particles are then said to be blocked. The blocking criterion for magnetic measurements at room temperature is defined, somewhat arbitrarily, as $\Delta / \mathrm{k}_{\mathrm{B}} T \approx 25$, corresponding to $\tau \approx 100 \mathrm{~s}$ and $\Delta \approx 1 \mathrm{eV}$ (see $>$ Chap. 20, "Magnetic Nanoparticles"). The 10-year stability criterion is $\Delta / \mathrm{k}_{\mathrm{B}} T \approx 40$. Cooling an ensemble of particles through the blocking temperature $T_{\mathrm{b}}=K_{\mathrm{u}} V / 25 \mathrm{k}_{\mathrm{B}}$ in a magnetic field leads to a relatively stable thermoremanent magnetization. The typical size of iron oxide particles that are superparamagnetic at room temperature is $\lesssim 10 \mathrm{~nm}$.

The magnetization of baked clay becomes blocked on cooling through $T_{\mathrm{b}}$ in the Earth's magnetic field. From the direction of the thermoremanent magnetization of appropriately dated hearths of pottery kilns, records of the historical secular variation of the Earth's field could be established, a topic known as archeomagnetism. Application of the same idea of thermoremanent magnetization to cooling of igneous rocks in the Earth's field provided a direct and convincing argument for geomagnetic reversals and continental drift; rocks cooling at different periods experienced fields of different polarities (Fig. 16), which followed an irregular sequence on a much longer timescale than the secular variation. The reversals could be dated using radioisotope methods on successive lava flows. This gave birth to the subfield of paleomagnetism and in turn allowed dating of the patterns of remanent magnetization picked up in oceanographic surveys conducted in the 1960s that established the reality of seafloor spreading. The theory of global plate tectonics has had far-reaching consequences for Earth science [29].

Superparamagnetic particles have found other practical uses. Ferrofluids, the colloidal suspensions of nanoparticles in oil or water with surfactants to inhibit agglomeration, are just one. They behave like anhysteretic ferromagnetic liquids. Individual particles or micron-sized polymer beads loaded with many of them may be functionalized with streptavidin and used as magnetic labels for specific biotin-tagged biochemical species, enabling them to be detected magnetically and separated by high-gradient magnetic separation based on the Kelvin force on a particle with moment $m, \boldsymbol{f}_{\mathrm{K}}=(m \cdot \nabla) \boldsymbol{B}$. Medical applications of magnetic fine particles include hyperthermia (targeted heating by exposure to a high-frequency magnetic field) and use as contrast agents in magnetic resonance imaging. However the most far-reaching application of magnetic nanoparticles so far has been in magnetic recording. 


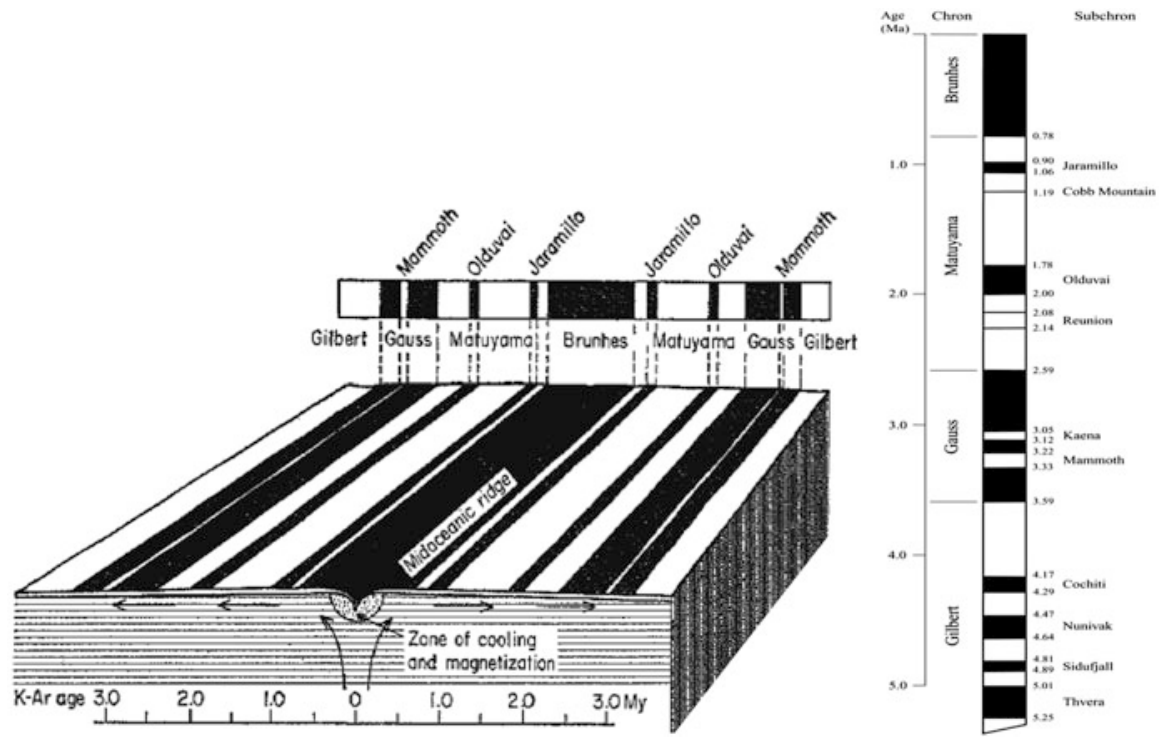

Fig. 16 Polarity of the thermoremanent magnetization measured across the floor of the Atlantic ocean (left). Current polarity is dark; reversed polarity is light. The pattern is symmetrical about the mid-ocean ridge, where new oceanic crust is being created. Random reversals of the Earth's field over the past $5 \mathrm{My}$, which are dated from other igneous lava flows, determine the chronological pattern (right) that is used to determine the rate of continental drift, of order centimeters per year. (McElhinney, Palaeomagnetism and Plate Tectonics [29], courtesy of Cambridge University Press)

\section{Magnetic Recording}

Particulate magnetic recording enjoyed a heyday that lasted over half a century, beginning with analog recording on magnetic tapes in Germany in the 1930s through digital recording on the hard and floppy discs that were introduced in the 1950s and 1960s, before eventually being superseded by thin-film recording in the late 1980 [27]. Particulate magnetic recording [30] was largely based on acicular particles of $\gamma \mathrm{Fe}_{2} \mathrm{O}_{3}$ often doped with 1-2\% Co. Elongated iron particles were also used, and acicular $\mathrm{CrO}_{2}$ was useful for rapid thermoremanent reproduction of videotapes on account of its low Curie temperature. Magnetic digital tape recording with hard ferrite particulate media continues to be used for archival storage.

The trend with magnetic media has always been to cram ever more digital data onto ever smaller areas. This has been possible because magnetic recording technology is inherently scaleable since reading is done by sensing the stray field of a patch of magnetized particles. It follows from Eq. 2 that since the dipole field decays as $1 / r^{3}$ and the moment $m \sim \boldsymbol{M} r^{3}$, the magnitude of $B$ is unchanged when everything else shrinks by the same scale factor - at least until the superparamagnetic limit $K V / \mathrm{k}_{\mathrm{B}} T \approx 40$ is reached, at which point the magnetic records become thermally unstable. To continue the scaling to bit sizes below 

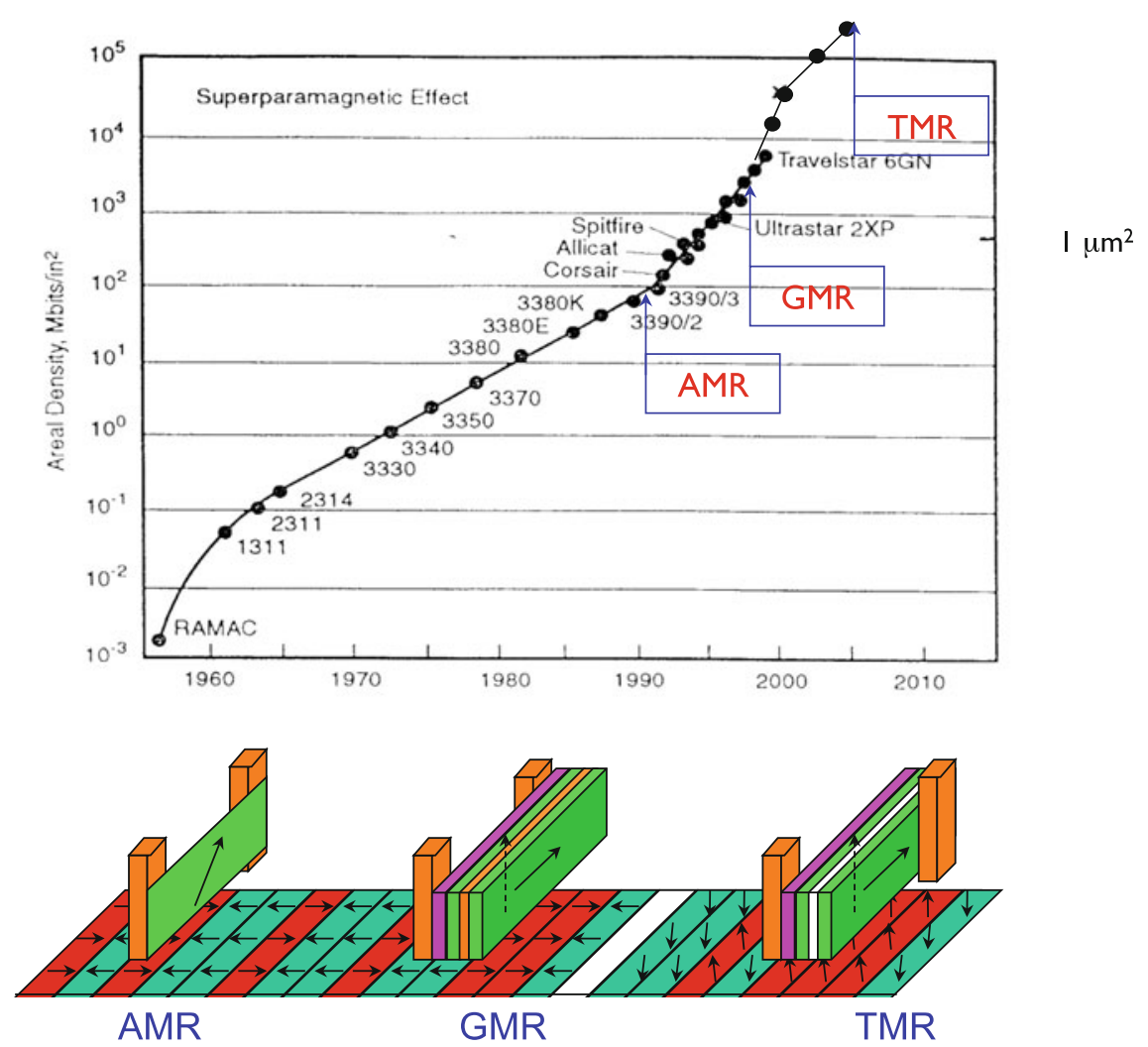

Fig. 17 Exponential growth of magnetic recording density over 50 years. The lower panel shows the magnetized magnetic medium with successive generations of read heads based on anisotropic magnetoresistance (AMR), giant magnetoresistance (GMR), and tunnel magnetoresistance (TMR)

$100 \mathrm{~nm}$, granular films of a highly anisotropic tetragonal Fe-Pt alloy are used to maintain stability of the magnetic records on ever-smaller oriented crystalline grains. The individual grains are less than $8 \mathrm{~nm}$ in diameter. Over the 65-year history of hard disc magnetic recording, the bit density has increased by eight orders of magnitude, at ever-decreasing cost (Fig. 17). Copies cost virtually nothing, and the volume of data stored on hard discs in computers and data centers doubles every year, so that as much new data is recorded each year as was ever recorded in all previous years of human history. This data explosion is unprecedented, and the third magnetic revolution, the big data revolution, is sure to have profound social and economic consequences. Although flash memory has displaced the magnetic hard discs from personal computers. The huge data centres, which are the physical embodiment of the 'cloud' where everything we download from the interenet is stored continue to use hard disc drives. 


\section{Methods of Investigation}

Magnetism is an experimental science, and progress in understanding and applications is generally contingent on advances in fabrication and measurement technology, whether it was fourteenth-century technology to fabricate a lodestone sphere or twenty-first-century technology to prepare and pattern a 16-layer thin-film stack for a magnetic sensor. The current phase of information technology relies largely on semiconductors to process digital data and on magnets for long-term storage.

For many physical investigations, magnetic materials are needed in special forms such as single crystals or thin films. Crystal growers have always been assiduously cultivated by neutron scatterers and other condensed matter physicists. Only with single crystals can tensor properties such as susceptibility, magnetostriction, and magnetotransport be measured properly. Nanoscale magnetic composites have extended the range of magnetic properties available in both hard and soft magnets. After 1970, thin-film growth facilities (sputtering, electron beam evaporation, pulsed laser deposition, molecular beam epitaxy) began to appear in magnetism laboratories worldwide. Ultra-high vacuum has facilitated the study of surface magnetism at the atomic level, while some of the motivation to investigate magneto-optics or magnetoresistance of metallic thin films, especially in thin-film heterostructures, arose from the prospect of massively improved magnetic data storage. Experimental methods are discussed in the chapters in Part 3 of this Handbook.

\section{Materials Preparation}

Silicon steel has been produced for electromagnetic applications by hot rolling since the beginning of the twentieth century. Annual production is now about 15 million tonnes, half of it in China. Permanent magnets, soft ferrites, and specialized magnetic alloys are produced in annual quantities ranging from upward of a hundred to a million tonnes. All such bulk applications of magnetism are highly sensitive to the cost of raw materials. This effectively disqualifies about a third of the elements in the periodic table and half of the heavy transition elements from consideration as alloy additives in bulk material. Newer methods such as mechanical alloying of elemental powders and rapid quenching from the melt by strip casting or melt spinning have joined the traditional methods of high-temperature furnace synthesis of bulk magnetic materials.

The transformation of magnetic materials science that has gathered pace since 1970 has been triggered by the ability to prepare new materials for magnetic devices in thin-film form. The minute quantity of material needed for a magnetic sensor or memory element, where the layers are tens of nanometers thick, means that any useful stable element can be considered. Platinum, for example, may sell for $\$ 30,000$ per kilogram, yet it is an indispensable constituent of the magnetic medium in the 400 million hard disc drives shipped each year that sell for about $\$ 60$ each. 
Uniform magnetic thin films down to atomic-scale thicknesses are produced in many laboratories by e-beam evaporation, sputtering, pulsed laser deposition, or molecular beam epitaxy, and the more complex tools needed to make patterned multilayer nanometer-scale thin-film stacks are quite widely available in research centers, as well as in the fabs of the electronics industry, which deliver the hardware on which the technology for modern life depends.

\section{Experimental Methods}

Advances in experimental observation underpin progress in conceptual understanding and technology. The discovery of magnetic resonance, the sharp absorption of microwave or radiofrequency radiation by Zeeman split levels of the magnetic moment of an atom or a nucleus in a magnetic field, or the collective precession of the entire magnetic moment of a solid was a landmark in modern magnetism. Significant mainly for the insight provided into solids and liquids at an atomic scale, electron paramagnetic resonance (EPR) was discovered by Yevgeny Zavoisky in 1944, and Felix Bloch and Edward Purcell established the existence of nuclear magnetic resonance (NMR) 2 years later. In 1958, Rudolf Mössbauer discovered a spectroscopic variant making use of low-energy gamma rays emitted by transitions from the excited states of some stable isotopes of iron $\left(\mathrm{Fe}^{57}\right)$ and certain rare earths $\left(\mathrm{Eu}^{151}\right.$, Dy ${ }^{161}$, etc.). All except Zavoisky received a Nobel Prize. The hyperfine interactions of the multipole moments of the nuclei (electric monopole, magnetic dipole, nuclear quadrupole) offered a point probe of electric and magnetic fields at the heart of the atom.

Larmor precession of the total magnetization of a ferromagnet in its internal field, usually in a resonant microwave cavity, was discussed theoretically by Landau and Evgeny Lifshitz in 1935, and ferromagnetic resonance (FMR) was confirmed experimentally 10 years later.

Of the non-resonant experimental probes, magnetic neutron scattering has probably been the most influential and generally useful. A beam of thermal neutrons from a nuclear reactor was first exploited for elastic diffraction in the USA in 1951 by Clifford Shull and Ernest Wohlan, who used the magnetic Bragg scattering to reveal the antiferromagnetic order in $\mathrm{MnO}$. Countless magnetic structures have been determined since, using the research reactors at Chalk River, Harwell, Brookhaven, Grenoble, and elsewhere. Magnetic excitations can be characterized by inelastic scattering of thermal neutrons, with the help of the triple-axis spectrometer developed in Canada by Bertram Brockhouse at Chalk River in 1956. Complete spin-wave dispersion relations provide a wealth of information on anisotropy and exchange. Newer accelerator-based neutron spallation sources at ISIS, Oak Ridge, and Lund provide intense pulses of neutrons by collision of highly energetic protons with a target of a heavy metal such as tungsten or mercury. They are most useful for studying magnetization dynamics. The low neutron scattering and absorption cross sections of most stable isotopes mean that neutrons can penetrate deeply into condensed matter. 
Besides neutrons, other intense beams of particles or electromagnetic radiation available at large-scale facilities have proved invaluable for probing magnetism. The intense, tunable ultraviolet and X-ray radiation from synchrotron sources allows the measurement of magnetic dichroism from deep atomic levels and permits the separate determination of spin and orbital contributions to the magnetic moment. The spectroscopy is element-specific and distinguishes different charge states of the same element. Spin-sensitive angular-resolved photoelectron spectroscopy makes it possible to map the spin-resolved electronic band structure. Muon methods are more specialized; they depend on the Larmor precession of short-lived ( $2.20 \mu \mathrm{s})$ positive muons when they are implanted into interstitial sites in a solid. Magnetic scattering methods are discussed in > Chap. 25, "Magnetic Scattering." The specialized instruments accessible at large-scale facilities supplement the traditional benchtop measurement capabilities of research laboratories.

Perhaps the most versatile and convenient of these, used to measure the magnetization and susceptibility of small samples, is the vibrating sample magnetometer invented by Simon Foner in 1956 and now a workhorse in magnetism laboratories across the world. The sample is vibrated in a uniform magnetic field, produced by an electromagnet or a superconducting coil, about the center of a set of quadrupole pickup coils, which provide a signal proportional to the magnetic moment. Since sample mass rather than sample volume is usually known, it is generally the mass susceptibility $\chi_{\mathrm{m}}=\chi / \rho$ that is determined.

Superconducting magnets now provide fields of up to 20 tesla or more for NMR and general laboratory use. The 5-10 T magnets are common, and they are usually cooled by closed-cycle cryocoolers to avoid wasting helium. Coupled with superconducting SQUID sensors, ultrasensitive magnetometers capable of measuring magnetic moments of $10^{-10} \mathrm{Am}^{2}$ or less are widely available. (The moment of a $5 \times \mathrm{mm}^{2}$ ferromagnetic monolayer is of order $10^{-8} \mathrm{Am}^{2}$.)

High magnetic fields, up to $35 \mathrm{~T}$, require expensive special installations with water-cooled Bitter magnets consuming many megawatts of electrical power. Resistive/superconducting hybrids in Tallahassee, Grenoble and Tsukuba, and Nijmegen can generate steady fields in excess of $40 \mathrm{~T}$. Higher fields imply short pulses; the higher the field, the shorter the pulse. Reusable coils generate pulsed fields approaching $100 \mathrm{~T}$ in Los Alamos, Tokyo, Dresden, Wuhan, and Toulouse.

Magnetic domain structures are usually imaged by magneto-optic Kerr microscopy, magnetic force microscopy, or scanning electron microscopy, although scanning SQUID and scanning Hall probe methods have also been developed. The Bitter method with a magnetite colloid continues to be used. All these methods image the surface or the stray field near the surface. Ultra-fast, picosecond magnetization dynamics are studied by optical pulse-probe methods based on the magneto-optic Kerr effect (MOKE). Transmission electron microscopy reveals the atomic structures of thin films and interfaces with atomic-scale resolution, while Lorentz microscopy offers magnetic contrast and holographic methods are able to image domains in three dimensions. Atomic-scale resolution can be achieved by point-probe methods with magnetic force microscopy or spin-polarized scanning tunnelling microscopy. The shift of focus in magnetism toward thin films and 
thin-film devices has been matched by the development of the sensitive analytical methods needed to characterize them. Hysteresis in thin films is conveniently measured by MOKE or by anomalous Hall effect (AHE) when the films are magnetized perpendicular to their plane. Magnetic fields and measurements are discussed in > Chap. 22, "Magnetic Fields and Measurements" and other chapters in Part 3.

An important consequence of the increasing availability of commercial superconducting magnets from the late 1960s was the development of medical diagnostic imaging of tissue based on proton relaxation times measured by NMR. Thousands of these scanners in hospitals across the world provide doctors with images of the hearts, brains, bones, and every sort of tumor.

\section{Computational Methods}

After about 1980, computer simulation began to emerge as a third force, besides experiment and theory, to gain insight into the physics of correlated electrons in magnetic systems. Contributions are mainly in two areas. One is calculation of the electronic structure, magnetic structure, magnetization, Curie temperature, and crystal structure of metallic alloys and compounds by using the density functional method. Magnetotransport in thin-film device structures can also be calculated. Here there is potential to seek and evaluate new magnetic phases in silico, before trying to make them in the laboratory. This magnetic genome program is in its infancy; success with magnetic materials to date has been limited, but the prospects are enticing.

The other area where computation has become a significant source of new insight is micromagnetic simulation. The domain structure and magnetization dynamics of magnetic thin-film structures and model heterostructures are intensely studied, both in industrial and academic laboratories. Simulation overcomes the surface limitation of experimental domain imaging. Software is generally based on finite element methods or the Landau-Lifshitz-Gilbert equation for magnetization dynamics.

\section{Spin Electronics}

As technology became available in the 1960s and 1970s to prepare high-quality metallic films with thicknesses in the nanometer range, interest in their magnetostansport properties grew. The terrain was being prepared for the emergence of a new phase of research that has grown to become the dominant theme in magnetism today - spin electronics. Spin electronics is the science of electron spin transport in solids. Many chapters in the Handbook deal with its various aspects.

For a long time, conventional electronics treated electrons simply as elementary Fermi-Dirac particles carrying a charge e, but it ignored their spin angular momentum $1 / 2 \hbar$. At first this was entirely justified; charge is conserved - the electron has no tendency to flip between states with charge \pm e, no matter how strongly 
it is scattered. But angular momentum is not conserved, and spin flip scattering is common in metals. Perhaps one scattering event in 100 changes the electron spin state, so the spin diffusion length $l_{\mathrm{S}}$ should be about ten times the mean free path $\lambda$ of the electron in a solid. When electronic device dimensions were many microns, there was no chance of an electron retaining the memory of any initial spin polarization it may have had, unless the device itself was ferromagnetic. Anisotropic magnetoresistance, where the scattering depends slightly on the relative orientation of the current and magnetization because of spin-orbit coupling, can be regarded as the archetypical spin electronic process. The relative magnitude of effect in permalloy, for instance, is only $\sim 2 \%$, but the alloy is extremely soft, on account of simultaneously vanishing anisotropy and magnetostriction, so a permalloy strip with current flowing at $45^{\circ}$ to the magnetic easy axis along the strip for maximum sensitivity - which can be achieved by a superposed "barber pole" pattern of highly conducting gold - makes a simple, miniature sensor for low magnetic fields, with a reasonable signal-to-noise ratio. AMR sensors replaced inductive sensors in the heads used to read data from hard discs in 1990, and the annual rate of increase of storage density improved sharply as a result.

Meanwhile, research activity on thin-film heterostructures where the layer thickness was comparable to the spin diffusion length began to pick up as more sophisticated thin-film vacuum deposition tools were developed. Spin diffusion lengths are $200 \mathrm{~nm}$ in $\mathrm{Cu}$, or about ten times the mean free path, as expected, but they are shorter in the ferromagnetic elements and sharply different for majorityand minority-spin electrons. The mean free path for minority-spin electrons in Co is only $1 \mathrm{~nm}$. Particularly influential and significant was the work carried out in 1988 in the groups of Peter Grunberg in Germany and Albert Fert in France on multilayer stacks of ferromagnetic and nonferromagnetic elements that led to the discovery of giant magnetoresistance (GMR). The effect depended on electrons retaining some of their spin polarization as they emerged from a ferromagnetic layer and crossed a nonmagnetic layer before reaching another ferromagnetic layer. Big changes of resistance were found when the relative alignment of the adjacent ferromagnetic iron layers in an $\mathrm{Fe}-\mathrm{Cr}$ multilayer stack was altered from antiparallel to parallel by applying a magnetic field (Fig. 18). At first, large magnetic fields and low temperatures were needed to see the resistance changes, but the structure was soon simplified to a sandwich of just two ferromagnetic layers with a copper spacer that became known as a spin valve. Spin valves worked at room temperature, and they were sensitive to the small stray fields produced by recorded magnetic tape or disc media. In order to make a useful sensor, it was necessary to pin the direction of magnetization of one of the ferromagnetic layers while leaving the other free to respond to an in-plane field (Fig. 19).

It was here that the phenomenon of exchange bias came to the rescue. First discovered in $\mathrm{Co} / \mathrm{CoO}$ core shell particles by Meiklejohn and Bean in 1956, it was extended to antiferromagnetic/ferromagnetic thin-film pairs in Néel's laboratory in Grenoble in the 1960s. By pinning one ferromagnetic layer with an adjacent antiferromagnet (initially $\mathrm{NiO}$ ), a useful GMR sensor could be produced with a magnetoresistance change of order $10 \%$. Exchange-biased GMR read heads 


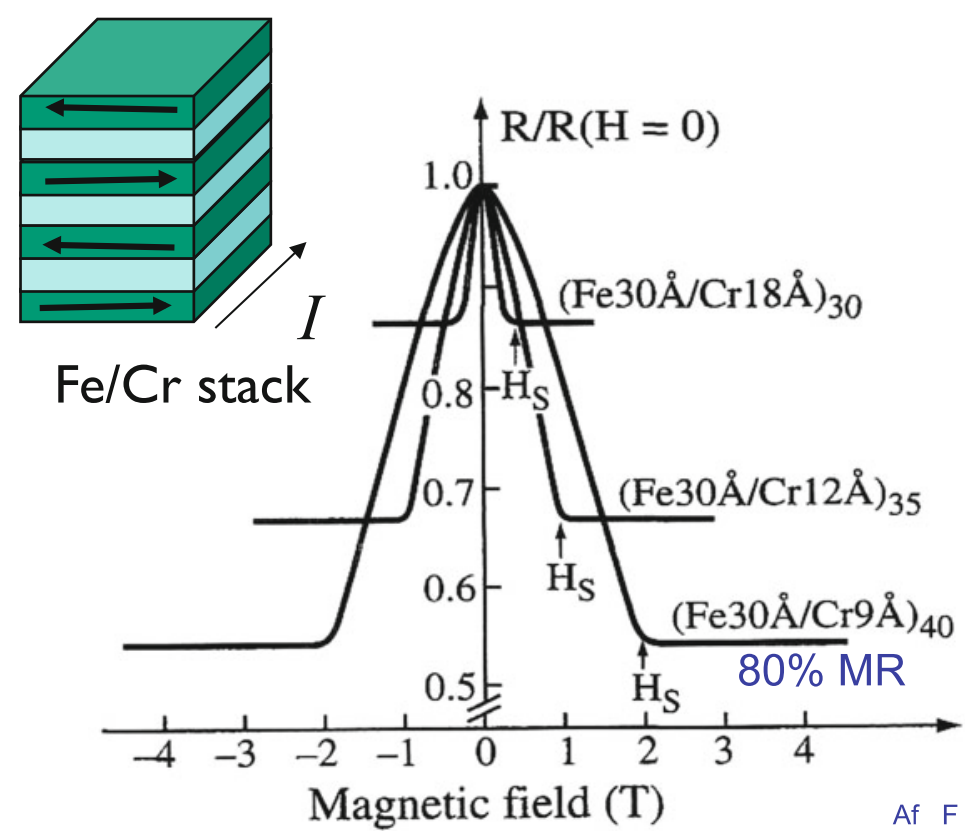

Fig. 18 Original measurement of giant magnetoresistance of a $\mathrm{FeCr}$ multilayer stack, where the iron layers naturally adopt an antiparallel conduction, which can be converted to a parallel configuration in an applied field [31]

developed by Stuart Parkin and colleagues went into production at IBM in 1998 - a remarkably rapid transfer from a laboratory discovery to mass production. Exchange bias was the first practical use of an antiferromagnet. The Nobel Physics Prize was awarded to Fert and Grunberg for their work in 2007.

Subsequent developments succeeded in eliminating the influence of the stray field of the pinned layer on the free layer by means of a synthetic antiferromagnet. This was another sandwich stack, like the slimmed-down spin valve, except the spacer was not copper, but an element that transferred exchange coupling from one ferromagnetic layer to the other. Ruthenium proved to be ideal, and a layer just $0.7 \mathrm{~nm}$ thick was found to be ideal for antiferromagnetic coupling [32].

GMR's tenure as read-head technology was to prove as short-lived as that of AMR. A new pretender with a much larger resistance change was based on the magnetic tunnel junction (MTJ), a modified spin valve where the nonmagnetic metal spacer is replaced by a thin layer of nonmagnetic insulator. Electron tunneling across an atomically thin vacuum barrier had been a striking prediction of quantum mechanics implicit in the idea of the wavefunction. The thin barrier was at first made of amorphous alumina, but it was replaced by crystalline $\mathrm{MgO}$ after it was found in 2004 that junctions where the $\mathrm{MgO}$ barrier acts as a spin filter exhibit tunneling magnetoresistance (TMR) in excess of 200\% [33, 34] (Fig. 19). The adoption of 


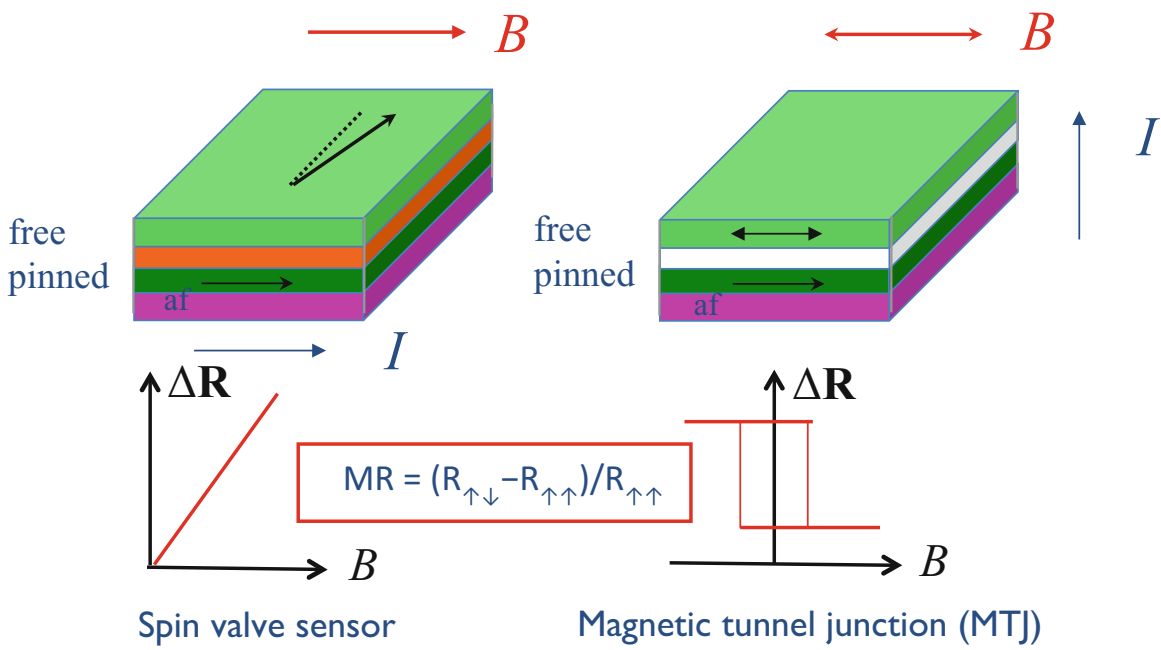

Fig. 19 Magnetic bilayer spin-valve stacks used as sensor (left) or as a memory element (right). In each case, the magnetization lies in-plane, and the lower ferromagnetic reference layer is pinned by exchange bias with the purple underlying antiferromagnetic layer, while the upper ferromagnetic free layer changes its orientation in response to the applied magnetic field. The change in stack resistance is plotted as a function of applied field. The magnetoresistance ratio MR is defined as the normalized resistance change between parallel and antiparallel orientation of the two ferromagnetic layers

TMR sensors in read heads in 2005 was accompanied by a change from in-plane to perpendicular recording on the magnetic medium.

Despite the changing generations of readers, the hard disc writer remained what is always had been, a miniature electromagnet that delivers sufficient flux to a patch of magnetic medium to overcome its coercivity and write the record. The extreme demands of magnetic recording have driven contactless magnetic sensing to new heights of sensitivity and miniaturization requiring increasingly hard magnetic media and new ways of writing them. Thin-film GMR and TMR structures have also taken a new life as magnetic switches for nonvolatile memory and logic. Most prominent is magnetic random access memory (MRAM), where huge arrays of memory cells are based on magnetic tunnel junctions. Magnetic sensing is discussed in $>$ Chaps. 31, "Magnetic Sensors," and $>22$, "Magnetic Fields and Measurements."

Magnetic thin-film technology has now advanced to the point where uniform layers in synthetic antiferromagnets and magnetic tunnel junctions only a few atoms thick are routinely deposited on entire 200 or $300 \mathrm{~mm}$ silicon wafers. A corollary of the short spin diffusion length of electrons in metals is the short distance - a few atomic monolayers - necessary for an electron to acquire spin polarization on transiting a ferromagnetic layer. Spin-polarized electron currents are central to spin electronics. 
The relation between magnetism and the angular momentum of electrons was unveiled in Larmor precession and the Einstein-de Haas experiment over a hundred years ago, but only in the present century has it become commonplace to associate electric currents with short-range flows of angular momentum. A spin-polarized current carrying its angular momentum into a ferromagnetic thin-film element can exert torque in two ways. It can create an effective magnetic field, causing Larmor precession of the magnetization of the element, and it can exert spin transfer torque, described by John Slonczewski in 1996 that counteracts damping of the precession and can be used to stabilize high-frequency oscillations or switch the magnetization without the need for an external magnetic field. Spin torque switching is effective for elements smaller than $100 \mathrm{~nm}$ in size, and unlike switching by current-induced "Oersted" fields, it is scalable - an essential requirement for electronic devices. Luc Berger showed that spin torque can also be used to manipulate domain walls.

A recurrent theme in the recent development of magnetism is the role of the spinorbit interaction. It is critically important in thin films [35], being responsible not only for the Kerr effect, magnetocrystalline anisotropy, and anisotropic magnetoresistance but also for the anomalous Hall effect and the spin Hall effect, whereby spin-orbit scattering of a current passing through a heavy metal or semiconductor produces a buildup of electrons with opposite spin on opposite sides of the conductor. This transverse spin current created by spin-orbit scattering enables the injection of angular momentum into an adjacent ferromagnetic layer and the change of its magnetization direction, an effect known as spin-orbit torque. Conversely, the inverse spin Hall effect is the appearance of a voltage across the heavy metal on pumping spin-polarized electrons into it from an adjacent ferromagnet, for example, by exciting ferromagnetic resonance.

The origin of the intrinsic anomalous Hall effect was an open question in magnetism, for well over a hundred years. A consensus is now building that it is due to the geometric Berry phase acquired by electrons moving adiabatically through a magnetic medium. The phase can be acquired from a non-collinear spin structure in real space or from topological singularities in the band sturcture in reciprocal space. Circular micromagnetic defects, known as skyrmions are also topologically protected.

Another manifestation of spin-orbit interaction is the Rashba effect; when an electric current is confined at an interface or surface, it tends to create a spin polarization normal to the direction of current flow. One of the most remarkable surface phenomena, arising from work by Haldane in 1988, is the possibility of topologically protected spin currents. A special feature of the band structure ensures that electrons at the surface or edges of some insulators or semiconductors are in gapless states. Electrons in these states can propagate around the surface without scattering, and they exhibit a spin order that winds around the surface as the direction of electron spin is usually locked at right angles to their linear momentum. Electrons at surfaces and interfaces can behave quite differently from electrons in the bulk, and interfaces are at the heart of electronic devices. The introduction of topological concepts into the discussion of spin-polarized electronic transport 
and magnetic defects is providing new insight into magnetism at the atomic and mesoscopic scales.

\section{Conclusion}

Magnetism since 1945 has been an area rich in discovery and useful applications, not least because of the tremendous increase in numbers of scientists and engineers working in the field. Magnet ownership for citizens of the developed world has skyrocketed from 1 or 2 magnets in 1945 to 100-200 60 years later or something of order a trillion if we count the individual magnetic bits on a hard disc in a desktop computer. Countless citizens throughout the world during this period already experienced magnetism's bounty at first hand in the form of a cassette tape recorder, and nowadays they can access the vast stores of magnetically recorded information in huge data centers via the Internet using a handheld device.

Magnetism is therefore playing a crucial role in the big data revolution that is engulfing us, by enabling the permanent data storage, from which we can make instant copies at practically no cost. It may deliver more nonvolatile computer memory if MRAM proves to a winning technology and possibly facilitate data transfer at rates up to the terahertz regime with the help of spin torque oscillators. There are potential magnetic solutions to the problems of ballooning energy consumption and the data rate bottleneck. There is potential to implement new paradigms for computation magnetically. While there is no certainty regarding the future form of information technology, improved existing solutions often have an inside track. Magnetism and magnetic materials may be a good bet.

There have been half a dozen paradigm shifts - radical changes in the ways of seeing and understanding the magnet and its magnetic field - during its 2000-year encounter with human curiosity. Implications of the big data revolution for human society are only beginning to come into focus, but they are likely to be as profound as on the previous two occasions when magnetism changed the world. This Handbook is a guide to what is going on.

Acknowledgments The author is grateful to Science Foundation Ireland for continued support, including contracts 10/IN.1/I3006, 13/ERC/I2561 and 16/IA/4534.

\section{Appendix: Units}

By the middle of the nineteenth century, it was becoming urgent to devise a standard set of units for electrical and magnetic quantities in order to exchange precise quantitative information. The burgeoning telegraph industry, for example, needed a standard of electrical resistance to control the quality of electrical cables. Separate electrostatic and electromagnetic unit systems based on the centimeter, the gram and the second had sprung into existence, and Maxwell and Jenkin proposed combining them in a coherent set of units in 1863. Their Gaussian cgs system was adopted 
internationally in 1881. Written in this unit system, Maxwell's equations relating electric and magnetic fields contain explicit factors of $c$, the velocity of light. Maxwell also introduced the idea of dimensional analysis in terms of the three basic quantities of mass, length, and time. The magnetic field $\boldsymbol{H}$ and the induction $\boldsymbol{B}$ are measured, respectively, in the numerically identical but dimensionally different units of oersted (Oe) and gauss (G).

Another basic unit, this time of electric current, was adopted in the Système International d'Unités (SI) in 1948. The number of basic units and dimensions in any system is an arbitrary choice; the SI (International System of Units) uses four insofar as we are concerned, the meter, kilogram, second, and ampere (or five if we include the mole). The system has been adopted worldwide for the teaching of science and engineering at school and universities; it embodies the familiar electrical units of volt, ampere, and ohm for electrical potential, current, and resistance. Maxwell's equations written in terms of two electric and two magnetic fields contain no factors of $c$ or $4 \pi$ in this system (Eq. 7), but they inevitably crop up elsewhere. $\boldsymbol{B}$ and $\boldsymbol{H}$ are obviously different quantities. The magnetic field strength $\boldsymbol{H}$, like the magnetization $\boldsymbol{M}$, has units of $\mathrm{Am}^{-1}$. The magnetic induction $\boldsymbol{B}$ is measured in tesla $(1 \mathrm{~T} \equiv$ $1 \mathrm{kgs}^{2} \mathrm{~A}^{-2}$ ). Magnetic moments have units of $\mathrm{Am}^{2}$, clearly indicating the origin of magnetism in electric currents and the absence of magnetic poles as real physical entities. The velocity of light is defined to be exactly $299,792,458 \mathrm{~ms}^{-1}$. The two constants $\mu_{0}$ and $\varepsilon_{0}$, the permeability and permittivity of free space, are related by $\mu_{0} \varepsilon_{0}=c^{2}$, where $\mu_{0}$ was $4 \pi 10^{-7} \mathrm{kgs}^{-2} \mathrm{~A}^{-2}$ according to the original definition of the ampere. However, in the new version of SI, which avoids the need for a physical standard kilogram, the equality of $\mu_{0}$ and $4 \pi 10^{-7}$ is not absolute, but it is valid to ten significant figures.

Only two of the three fields $\boldsymbol{B}, \boldsymbol{H}$, and $\boldsymbol{M}$ are independent (Fig. 4). The relation between them is Eq. 8, $\boldsymbol{B}=\mu_{0}(\boldsymbol{H}+\boldsymbol{M})$. This is the Sommerfeld convention for SI. The alternative Kenelly convention, often favored by electrical engineers, defines magnetic polarization as $\boldsymbol{J}=\mu_{0} \boldsymbol{M}$, so that the relation becomes $\boldsymbol{B}=\mu_{0} \boldsymbol{H}+\boldsymbol{J}$. We

Table 1 Numerical conversion factors between SI and cgs units

\begin{tabular}{|c|c|c|c|}
\hline Physical quantity & Symbol & SI to cgs conversion & cgs to SI conversion \\
\hline $\begin{array}{l}B \text {-field (magnetic flux } \\
\text { density) }\end{array}$ & $B$ & 1 tesla $=10$ kilogauss & $\begin{array}{l}1 \text { gauss } *=0.1 \\
\text { millitesla }\end{array}$ \\
\hline $\begin{array}{l}H \text {-field (magnetic field } \\
\text { intensity) }\end{array}$ & $H$ & $1 \mathrm{kAm}^{-1}=12.57$ oersted & $\begin{array}{l}1 \text { oersted }^{\S}=79.58 \\
\mathrm{Am}^{-1}\end{array}$ \\
\hline Magnetic moment & $m$ & $1 \mathrm{Am}^{2}=1000 \mathrm{emu}$ & $1 \mathrm{emu}=1 \mathrm{mAm}^{2}$ \\
\hline Magnetization & $M$ & $1 \mathrm{Am}^{-1}=12.57$ gauss $^{\dagger}$ & 1 gauss $^{\dagger}=79.58 \mathrm{Am}^{-1}$ \\
\hline Specific magnetization & $\sigma$ & $1 \mathrm{Am}^{2} \mathrm{~kg}^{-1}=1 \mathrm{emu} \mathrm{g}^{-1}$ & $\begin{array}{l}1 \mathrm{emu} \mathrm{g}^{-1}=1 \\
\mathrm{Am}^{2} \mathrm{~kg}^{-1}\end{array}$ \\
\hline Magnetic energy density & $(B H)$ & $1 \mathrm{kJm}^{-3}=0.1257 \mathrm{MGOe}$ & $1 \mathrm{MGOe}=7.96 \mathrm{kJm}^{-3}$ \\
\hline $\begin{array}{l}\text { Dimensionless } \\
\text { susceptibility } M / H\end{array}$ & $x$ & $1(\mathrm{SI})=1 / 4 \pi(\mathrm{cgs})$ & $1(\mathrm{cgs})=4 \pi(\mathrm{SI})$ \\
\hline
\end{tabular}

*symbol G; ${ }^{\S}$ symbol Oe; ${ }^{\dagger} 4 \pi M$; Note: $12.57=4 \pi ; 79.58=1000 / 4 \pi$ 
follow the Sommerfeld convention in this Handbook. The magnetic field strength $H$ is not measured in units of Tesla in any generally accepted convention, but it can be so expressed by multiplying by $\mu_{0}$.

At the present time, Gaussian cgs units remain in widespread use in research publications, despite the obvious advantages of SI. The use of the cgs system in magnetism runs into the difficulty that units of $\boldsymbol{B}$ and $\boldsymbol{H}, \mathrm{G}$ and Oe, are dimensionally different but numerically the same; $\mu_{0}=1$, but it normally gets left out of the equations, which makes it impossible to check whether the dimensions balance. Table 1 lists the conversion factors and units in the two systems. The cgs equivalent of Eq. 8 is $\boldsymbol{B}=\boldsymbol{H}+4 \pi \boldsymbol{M}$. The cgs unit of charge is defined in such a way that $\varepsilon_{0}=1 / 4 \pi \mathrm{c}$ and $\mu_{0}=4 \pi / \mathrm{c}$ so factors of c appear in Maxwell's equations in place of the electric and magnetic constants. Convenient numerical conversion factors between the two systems of units are provided in Table 1 .

Theoretical work in magnetism is sometimes presented in a set of units where $c=\hbar=\mathrm{k}_{\mathrm{B}}=1$. This simplifies the equations, but does nothing to facilitate quantitative comparison with experimental measurements.

\section{References}

1. Kloss, A.: Geschichte des Magnetismus. VDE-Verlag, Berlin (1994)

2. Matthis, D.C.: Theory of Magnetism, ch. 1. Harper and Row, New York (1965)

3. Needham, J.: Science and Civilization in China, vol. 4, part 1. Cambridge University Press, Cambridge (1962)

4. Schmid, P.A.: Two early Arabic sources of the magnetic compass. J. Arabic Islamic Studies. 1, 81-132 (1997)

5. Fowler, T.: Bacon's Novum Organum. Clarendon Press, Oxford (1878)

6. Pierre Pèlerin de Maricourt: The Letter of Petrus Peregrinus on the Magnet, AD 1292, Trans. Br. Arnold. McGraw-Hill, New York (1904)

7. Gilbert, W: De Magnete, Trans. P F Mottelay. Dover Publications, New York (1958)

8. Fara, P.: Sympathetic Attractions: Magnetic Practices, Beliefs and Symbolism in EighteenthCentury England. Princeton University Press, Princeton (1996)

9. Mottelay, P.F.: Bibliographical History of Electricity and Magnetism. Arno Press, New York (1975)

10. Faraday, M.: Experimental Researches in Electricity, volume III. Bernard Quartrich, London (1855)

11. Maxwell, J.C.: A Treatise on Electricity and Magnetism, two volumes. Clarendon Press, Oxford (1873) (Reprinted Cambridge University Press, 2010)

12. Bertotti, G.: Hysteresis in Magnetism. Academic Press, New York (1998)

13. Brown, W.F.: Micromagnetics. Interscience, New York (1963)

14. Sato, M., Ishii, Y.: Simple and approximate expressions of demagnetizing factors of uniformly magnetized rectangular rod and cylinder. J. Appl. Phys. 66, 983-988 (1989)

15. Hunt, B.J.: The Maxwellians. Cornell University Press, New York (1994)

16. Joule, J.P.: On the Effects of Magnetism upon the Dimensions of Iron and Steel Bars. Philosoph. Mag. Third Series. 76-87, 225-241 (1847)

17. Thomson, W.: On the electrodynamic qualities of metals. Effects of magnetization on the electric conductivity of nickel and iron. Proc. Roy. Soc. 8, 546-550 (1856)

18. Kerr, J.: On rotation of the plane of the polarization by reflection from the pole of a magnet. Philosoph. Mag. 3, 321 (1877) 
19. Ewing, J.A.: Magnetic Induction in Iron and Other Metals, 3rd edn. The Electrician Publishing Company, London (1900)

20. Tomonaga, S.: The Story of Spin. University of Chicago Press, Chicago (1974)

21. Marage, P., Wallenborn, G. (eds.): Les Conseils Solvay et Les Débuts de la Physique Moderne. Université Libre de Bruxelles (1995)

22. Ballhausen, C.J.: Introduction to Ligand Field Theory. McGraw Hill, New York (1962)

23. Bozorth, R.M.: Ferromagnetism. McGraw Hill, New York (1950) (reprinted Wiley - IEEE Press, 1993)

24. Goodenough, J.B.: Magnetism and the Chemical Bond. Interscience, New York (1963)

25. Smit, J., Wijn, H.P.J.: Ferrites; Physical Properties of Ferrrimagnetic Oxides. Philips Technical Library, Eindhoven (1959)

26. Coey, J.M.D., Viret, M., von Molnar, S.: Mixed valence manganites. Adv. Phys. 48, 167 (1999)

27. Wang, S.X., Taratorin, A.M.: Magnetic Information Storage Technology. Academic Press, San Diego (1999)

28. Coey, J.M.D. (ed.): Rare-Earth Iron Permanent Magnets. Clarendon Press, Oxford (1996)

29. McElhinney, M.W.: Palaeomagnetism and Plate Tectonics. Cambridge University Press (1973)

30. Daniel, E.D., Mee, C.D., Clark, M.H. (eds.): Magnetic Recording, the First Hundred Years. IEEE Press, New York (1999)

31. Baibich, M.N., Broto, J.M., Fert, A., Nguyen Van Dau, F., et al.: Giant magnetoresistance of (001) $\mathrm{Fe} /(001) \mathrm{Cr}$ magnetic superlattices. Phys. Rev. Lettters. 61, 2472 (1988)

32. Parkin, S.S.P.: Systematic variation on the strength and oscillation period of indirect magnetic exchange coupling through the 3d, 4d and 5d transition metals. Phys. Rev. B. 67, 3598 (1991)

33. Parkin, S.S.P., Kaiser, C., Panchula, A., Rice, P.M., Hughes, B., et al.: Giant tunneling magnetoresistance with $\mathrm{MgO}$ (100) tunnel barriers. Nat. Mater. 3, 862-867 (2004)

34. Yuasa, S., Nagahama, T., Fukushima, A., Suzuki, Y., Ando, K.: Giant room-temperature magnetoresistance in single-crystal $\mathrm{Fe} / \mathrm{MgO} / \mathrm{Fe}$ magnetic tunnel junctions. Nat. Mater. 3, 868-871 (2004)

35. Sinova, J., Valenzuela, S.O., Wunderlich, J., Bach, C.H., Wunderlich, J.: Spin hall effects. Rev. Mod. Phys. 87, 1213 (2015)

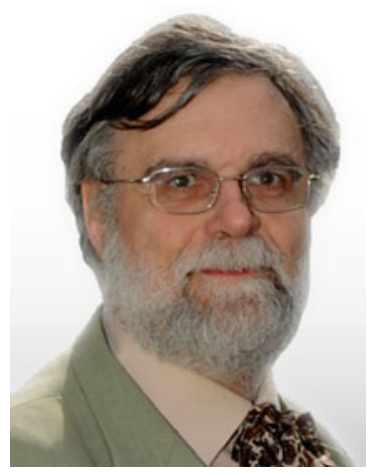

Michael Coey received his $\mathrm{PhD}$ from the University of Manitoba in 1971; he has worked at the CNRS, Grenoble, IBM, Yorktown Heights, and, since 1979, Trinity College Dublin. Author of several books and many papers, his interests include amorphous and disordered magnetic materials, permanent magnetism, oxides and minerals, $d^{0}$ magnetism, spin electronics, magnetoelectrochemistry, magnetofluidics, and the history of ideas. 